\title{
On Symmetry and Conserved Quantities in Classical Mechanics
}

\author{
J. Butterfield ${ }^{1}$
}

All Souls College

Oxford OX1 4AL

Tuesday 12 July 2005; for a Festschrift for Jeffrey Bub, ed. W. Demopoulos and I. Pitowsky, Kluwer: University of Western Ontario Series in Philosophy of Science. ${ }^{2}$

\begin{abstract}
This paper expounds the relations between continuous symmetries and conserved quantities, i.e. Noether's "first theorem", in both the Lagrangian and Hamiltonian frameworks for classical mechanics. This illustrates one of mechanics' grand themes: exploiting a symmetry so as to reduce the number of variables needed to treat a problem.

I emphasise that, for both frameworks, the theorem is underpinned by the idea of cyclic coordinates; and that the Hamiltonian theorem is more powerful. The Lagrangian theorem's main "ingredient", apart from cyclic coordinates, is the rectification of vector fields afforded by the local existence and uniqueness of solutions to ordinary differential equations. For the Hamiltonian theorem, the main extra ingredients are the asymmetry of the Poisson bracket, and the fact that a vector field generates canonical transformations iff it is Hamiltonian.
\end{abstract}

\footnotetext{
${ }^{1}$ email: jb56@cus.cam.ac.uk; jeremy.butterfield@all-souls.oxford.ac.uk

${ }^{2}$ It is a pleasure to dedicate this paper to Jeff Bub, who has made such profound contributions to the philosophy of quantum theory. Though the paper is about classical, not quantum, mechanics, I hope that with his love of geometry, he enjoys symplectic forms as much as inner products!
} 


\section{Contents}

1 Introduction 3

2 Lagrangian mechanics 4

2.1 Lagrange's equations . . . . . . . . . . . . . . . . . . . . 4

2.2 Geometrical perspective . . . . . . . . . . . . . 7

2.2.1 Some restrictions of scope . . . . . . . . . . . 7

2.2.2 The tangent bundle . . . . . . . . . . . . . . 8

3 Noether's theorem in Lagrangian mechanics 10

3.1 Preamble: a modest plan . . . . . . . . . . . . . . . . . . . . . . . 10

3.2 Vector fields and symmetries-variational and dynamical . . . . . . . . 12

3.2.1 Vector fields on $T Q$; lifting fields from $Q$ to $T Q \ldots 13$

3.2.2 The definition of variational symmetry . . . . . . . . . . . . . 14

3.2.3 A contrast with dynamical symmetries . . . . . . . . . . . . . 14

3.3 The conjugate momentum of a vector field . . . . . . . . . . . . . . 17

3.4 Noether's theorem; and examples . . . . . . . . . . . . . . . . . . 17

3.4.1 A geometrical formulation . . . . . . . . . . . . . 20

4 Hamiltonian mechanics introduced $\quad 21$

4.1 Preamble . . . . . . . . . . . . . . . . . . . . . . 21

4.2 Hamilton's equations . . . . . . . . . . . . . . . . . 22

4.2.1 The equations introduced . . . . . . . . . . . . 22

4.2.2 Cyclic coordinates in the Hamiltonian framework . . . . . . 23

4.2.3 The Legendre transformation and variational principles . . . . . 25

4.3 Symplectic forms on vector spaces . . . . . . . . . . . . . . 25

4.3.1 Time-evolution from the gradient of $H \ldots \ldots$. . . . . . . 26

4.3.2 Interpretation in terms of areas . . . . . . . . . . . . . 26

4.3.3 Bilinear forms and associated linear maps . . . . . . . . . . . 28

5 Poisson brackets and Noether's theorem 33

5.1 Poisson brackets introduced . . . . . . . . . . . . . . . . . 33

5.2 Hamiltonian vector fields . . . . . . . . . . . . . . . . . . 35 
5.3 Noether's theorem . . . . . . . . . . . . . . . . . . 36

5.3.1 An apparent "one-liner", and three claims . . . . . . . . . . 36

5.3.2 The relation to the Lagrangian version . . . . . . . . . . . . . . 38

5.4 Glimpsing the "complete solution" . . . . . . . . . . . . . . . . . 40

$\begin{array}{lll}6 & \text { A geometrical perspective } & 41\end{array}$

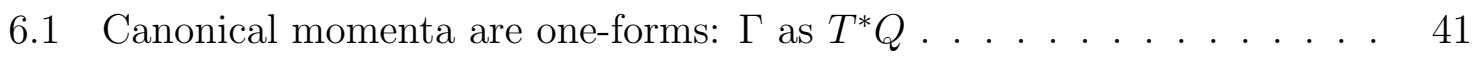

6.2 Forms, wedge-products and exterior derivatives . . . . . . . . . 43

6.2.1 The exterior algebra; wedge-products and contractions . . . . . 43

6.2.2 Differential forms; the exterior derivative; the Poincaré Lemma . 45

6.3 Symplectic manifolds; the cotangent bundle as a symplectic manifold . 46

6.3.1 Symplectic manifolds . . . . . . . . . . . . . . . . 47

6.3.2 The cotangent bundle . . . . . . . . . . . . . . . 48

6.4 Geometric formulations of Hamilton's equations . . . . . . . . . . . . . 49

6.5 Noether's theorem completed . . . . . . . . . . . . . . . 50

6.6 Darboux's theorem, and its role in reduction . . . . . . . . . . . . . 51

6.7 Geometric formulation of the Legendre transformation . . . . . . . . . 53

6.8 Glimpsing the more general framework of Poisson manifolds . . . . . . 55

$\begin{array}{lll}7 & \text { References } & 57\end{array}$ 


\section{Introduction}

The strategy of simplifying a mechanical problem by exploiting a symmetry so as to reduce the number of variables is one of classical mechanics' grand themes. It is theoretically deep, practically important, and recurrent in the history of the subject. Indeed, it occurs already in 1687, in Newton's solution of the Kepler problem; (or more generally, the problem of two bodies exerting equal and opposite forces along the line between them). The symmetries are translations and rotations, and the corresponding conserved quantities are the linear and angular momenta.

This paper will expound one central aspect of this large subject. Namely, the relations between continuous symmetries and conserved quantities - in effect, Noether's "first theorem": which I expound in both the Lagrangian and Hamiltonian frameworks, though confining myself to finite-dimensional systems. As we shall see, this topic is underpinned by the theorems in elementary Lagrangian and Hamiltonian mechanics about cyclic (ignorable) coordinates and their corresponding conserved momenta. (Again, there is a glorious history: these theorems were of course clear to these subjects' founders.) Broadly speaking, my discussion will make increasing use, as it proceeds, of the language of modern geometry. It will also emphasise Hamiltonian, rather than Lagrangian, mechanics: apart from mention of the Legendre transformation, the Lagrangian framework drops out wholly after Section 3.4.1. ${ }^{3}$

There are several motivations for studying this topic. As regards physics, many of the ideas and results can be generalized to infinite-dimensional classical systems; and in either the original or the generalized form, they underpin developments in quantum theories. The topic also leads into another important subject, the modern theory of symplectic reduction: (for a philosopher's introduction, cf. Butterfield (2006)). As regards philosophy, the topic is a central focus for the discussion of symmetry, which is both a long-established philosophical field and a currently active one: cf. Brading and Castellani (2003). (Some of the current interest relates to symplectic reduction, whose philosophical significance has been stressed recently, especially by Belot: Butterfield (2006) gives references.)

The plan of the paper is as follows. In Section 2, I review the elements of the Lagrangian framework, emphasising the elementary theorem that cyclic coordinates yield conserved momenta, and introducing the modern geometric language in which mechanics is often cast. Then I review Noether's theorem in the Lagrangian framework (Section 3). I emphasise how the theorem depends on two others: the elementary theorem about cyclic coordinates, and the local existence and uniqueness of solutions of ordinary differential equations. Then I introduce Hamiltonian mechanics, again emphasising how cyclic coordinates yield conserved momenta; and approaching canonical transformations through the symplectic form (Section 4). This leads to Section 5's discussion of Poisson brackets; and thereby, of the Hamiltonian version of Noether's

\footnotetext{
${ }^{3}$ It is worth noting the point, though I shall not exploit it, that symplectic structure can be seen in the classical solution space of the Lagrangian framework; cf. (3) of Section 6.7.
} 
theorem. In particular, we see what it would take to prove that this version is more powerful than (encompasses) the Lagrangian version. By the end of the Section, it only remains to show that a vector field generates a one-parameter family of canonical transformations iff it is a Hamiltonian vector field. It turns out that we can show this without having to develop much of the theory of canonical transformations. We do so in the course of the final Section's account of the geometric structure of Hamiltonian mechanics, especially the symplectic structure of a cotangent bundle (Section 6). Finally, we end the paper by mentioning a generalized framework for Hamiltonian mechanics which is crucial for symplectic reduction. This framework takes the Poisson bracket, rather than the symplectic form, as the basic notion; with the result that the state-space is, instead of a cotangent bundle, a generalization called a 'Poisson manifold'.

\section{Lagrangian mechanics}

\subsection{Lagrange's equations}

We consider a mechanical system with $n$ configurational degrees of freedom (for short: $n$ freedoms), described by the usual Lagrange's equations. These are $n$ second-order ordinary differential equations:

$$
\frac{d}{d t}\left(\frac{\partial L}{\partial \dot{q}^{i}}\right)-\frac{\partial L}{\partial q^{i}}=0, \quad i=1, \ldots, n ;
$$

where the Lagrangian $L$ is the difference of the kinetic and potential energies: $L:=$ $K-V$. (We use $K$ for the kinetic energy, not the traditional $T$; for in differential geometry, we will use $T$ a lot, both for 'tangent space' and 'derivative map'.)

I should emphasise at the outset that several special assumptions are needed in order to deduce eq. 2.1 from Newton's second law, as applied to the system's component parts: (assumptions that tend to get forgotten in the geometric formulations that will dominate later Sections!) But I will not go into many details about this, since:

(i): there is no single set of assumptions of mimimum logical strength (nor a single "best package-deal" combining simplicity and mimimum logical strength);

(ii): full discussions are available in many textbooks (or, from a philosophical viewpoint, in Butterfield 2004a: Section 3).

I will just indicate a simple and commonly used sufficient set of assumptions. But owing to (i) and (ii), the details here will not be cited in later Sections.

Note first that if the system consists of $N$ point-particles (or bodies small enough to be treated as point-particles), so that a configuration is fixed by $3 N$ cartesian coordinates, we may yet have $n<3 N$. For the system may be subject to constraints and we will require the $q^{i}$ to be independently variable. More specifically, let us assume that any constraints on the system are holonomic; i.e. each is expressible as an equation $f\left(r^{1}, \ldots, r^{m}\right)=0$ among the coordinates $r^{k}$ of the system's component parts; (here the 
$r^{k}$ could be the $3 N$ cartesian coordinates of $N$ point-particles, in which case $\left.m:=3 N\right)$. A set of $c$ such constraints can in principle be solved, defining a $(m-c)$-dimensional hypersurface $Q$ in the $m$-dimensional space of the $r$ s; so that on the configuration space $Q$ we can define $n:=m-c$ independent coordinates $q^{i}, i=1, \ldots, n$.

Let us also assume that any constraints on the system are: (i) scleronomous, i.e. independent of time, so that $Q$ is identified once and for all; (ii) ideal, i.e. the forces that maintain the constraints would do no work in any possible displacement consistent with the constraints and applied forces (a 'virtual displacement'). Let us also assume that the forces applied to the system are monogenic: i.e. the total work $\delta w$ done in an infinitesimal virtual displacement is integrable; its integral is the work function $U$. (The term 'monogenic' is due to Lanczos (1986, p. 30), but followed by others e.g. Goldstein et al. (2002, p. 34).) And let us assume that the system is conservative: i.e. the work function $U$ is independent of both the time and the generalized velocities $\dot{q}_{i}$, and depends only on the $q^{i}: U=U\left(q^{1}, \ldots, q^{n}\right)$.

So to sum up: let us assume that the constraints are holonomic, scleronomous and ideal, and that the system is monogenic with a velocity-independent work-function. Now let us define $K$ to be the kinetic energy; i.e. in cartesian coordinates, with $k$ now labelling particles, $K:=\Sigma_{k} \frac{1}{2} m_{k} \mathbf{v}_{k}^{2}$. Let us also define $V:=-U$ to be the potential energy, and set $L:=K-V$. Then the above assumptions imply eq. 2.1. ${ }^{4}$

To solve mechanical problems, we need to integrate Lagrange's equations. Recall the idea from elementary calculus that $n$ second-order ordinary differential equations have a (locally) unique solution, once we are given $2 n$ arbitrary constants. Broadly speaking, this idea holds good for Lagrange's equations; and the $2 n$ arbitrary constants can be given just as one would expect - as the initial configuration and generalized velocities $q^{i}\left(t_{0}\right), \dot{q}^{i}\left(t_{0}\right)$ at time $t_{0}$. More precisely: expanding the time derivatives in eq. 2.1 , we get

$$
\frac{\partial^{2} L}{\partial \dot{q}^{j} \partial \dot{q}^{i}} \ddot{q}^{j}=-\frac{\partial^{2} L}{\partial q^{j} \partial \dot{q}^{i}} \dot{q}^{j}-\frac{\partial^{2} L}{\partial t \partial \dot{q}^{i}}+\frac{\partial L}{\partial \dot{q}^{i}}
$$

so that the condition for being able to solve these equations to find the accelerations at some initial time $t_{0}, \ddot{q}^{i}\left(t_{0}\right)$, in terms of $q^{i}\left(t_{0}\right), \dot{q}^{i}\left(t_{0}\right)$ is that the Hessian matrix $\frac{\partial^{2} L}{\partial \dot{q}^{i} \partial \dot{q}^{j}}$ be nonsingular. Writing the determinant as $\mid$ |, and partial derivatives as subscripts, the condition is that:

$$
\left|\frac{\partial^{2} L}{\partial \dot{q}^{j} \partial \dot{q}^{i}}\right| \equiv\left|L_{\dot{q}^{j} \dot{q}^{i}}\right| \neq 0
$$

This Hessian condition holds in very many mechanical problems; and henceforth, we assume it. (If it fails, we enter the territory of constrained dynamics; for which cf. e.g. Henneaux and Teitelboim (1992, Chapters 1-5).) It underpins most of what follows: for it is needed to define the Legendre transformation, by which we pass from Lagrangian

\footnotetext{
${ }^{4}$ Though I shall not develop any details, there is of course a rich theory about these and related assumptions. One example, chosen with an eye to our later use of geometry, is that assuming scleronomous constraints, $K$ is readily shown to be a homogeneous quadratic form in the generalized velocities, i.e. of the form $K=\sum_{i, j}^{n} a_{i j} \dot{q}^{i} \dot{q}^{j}$; and so $K$ defines a metric on the configuration space.
} 
to Hamiltonian mechanics.

Of course, even with eq. 2.3, it is still in general hard in practice to solve for the $\ddot{q}^{i}\left(t_{0}\right)$ : they are buried in the lhs of eq. 2.2. In (5) of Section 2.2.2, this will motivate the move to Hamiltonian mechanics. ${ }^{5}$

Given eq. 2.3, and so the accelerations at the initial time $t_{0}$, the basic theorem on the (local) existence and uniqueness of solutions of ordinary differential equations can be applied. (We will state this theorem in Section 3.4 in connection with Noether's theorem.)

By way of indicating the rich theory that can be built from eq. 2.1 and 2.3, I mention one main aspect: the power of variational formulations. Eq. 2.1 are the Euler-Lagrange equations for the variational problem $\delta \int L d t=0$; i.e. they are necessary and sufficient for the action integral $I=\int L d t$ to be stationary. But variational principles will play no further role in this paper; (Butterfield 2004 is a philosophical discussion).

But our main concern, here and throughout this paper, is how symmetries yield conserved quantities, and thereby reduce the number of variables that need to be considered in solving a problem. In fact, we are already in a position to prove Noether's theorem, to the effect that any (continuous) symmetry of the Lagrangian $L$ yields a conserved quantity. But we postpone this to Section 3, until we have developed some more notions, especially geometric ones.

We begin with the idea of generalized momenta, and the result that the generalized momentum of any cyclic coordinate is a constant of the motion: though very simple, this result is the basis of Noether's theorem. Elementary examples prompt the definition of the generalized, or canonical, momentum, $p_{i}$, conjugate to a coordinate $q^{i}$ as: $\frac{\partial L}{\partial \dot{q}^{i}}$; (this was first done by Poisson in 1809). Note that $p_{i}$ need not have the dimensions of momentum: it will not if $q^{i}$ does not have the dimension length. So Lagrange's equations can be written:

$$
\frac{d}{d t} p_{i}=\frac{\partial L}{\partial q^{i}}
$$

We say a coordinate $q^{i}$ is cyclic if $L$ does not depend on $q^{i}$. (The term comes from the example of an angular coordinate of a particle subject to a central force. Another term is: ignorable.) Then the Lagrange equation for a cyclic coordinate, $q^{n}$ say, becomes $\dot{p}_{n}=0$, implying

$$
p_{n}=\text { constant, } c_{n} \text { say. }
$$

So: the generalized momentum conjugate to a cyclic coordinate is a constant of the motion.

It is straightforward to show that this simple result encompasses the elementary theorems of the conservation of momentum, angular momentum and energy: this last corresponding to time's being a cyclic coordinate. As a simple example, consider the

\footnotetext{
${ }^{5}$ This is not to say that Hamiltonian mechanics makes all problems "explicitly soluble": if only! For a philosophical discussion of the various meanings of 'explicit solution', cf. Butterfield (2004a: Section 2.1).
} 
angular momentum of a free particle. The Lagrangian is, in spherical polar coordinates,

$$
L=\frac{1}{2} m\left(\dot{r}^{2}+r^{2} \dot{\theta}^{2}+r^{2} \dot{\phi}^{2} \sin ^{2} \theta\right)
$$

so that $\partial L / \partial \phi=0$. So the conjugate momentum

$$
\frac{\partial L}{\partial \dot{\phi}}=m r^{2} \dot{\phi} \sin ^{2} \theta
$$

which is the angular momentum about the $z$-axis, is conserved.

\subsection{Geometrical perspective}

\subsubsection{Some restrictions of scope}

I turn to give a brief description of the elements of Lagrangian mechanics in terms of modern differential geometry. Here 'brief' indicates that:

(i): I will assume without explanation various geometric notions, in particular: manifold, vector, 1-form (covector), metric, Lie derivative and tangent bundle.

(ii): I will disregard issues about degrees of smoothness: all manifolds, scalars, vectors etc. will be assumed to be as smooth as needed for the context.

(iii): I will also simplify by speaking "globally, not locally". I will speak as if the scalars, vector fields etc. are defined on a whole manifold; when in fact all that we can claim in application to most systems is a corresponding local statement-because for example, differential equations are guaranteed the existence and uniqueness only of a local solution. ${ }^{6}$

We begin by assuming that the configuration space (i.e. the constraint surface) $Q$ is a manifold. The physical state of the system, taken as a pair of configuration and generalized velocities, is represented by a point in the tangent bundle $T Q$ (also known as 'velocity phase space'). That is, writing $T_{x}$ for the tangent space at $x \in Q, T Q$ has points $(x, \tau), x \in Q, \tau \in T_{x}$. We will of course often work with the natural coordinate systems on $T Q$ induced by coordinate systems $q$ on $Q$; i.e. with the $2 n$ coordinates $(q, \dot{q}) \equiv\left(q^{i}, \dot{q}^{i}\right)$.

The main idea of the geometric perspective is that this tangent bundle is the arena for Lagrangian mechanics. So various previous notions and results are now expressed in terms of the tangent bundle. In particular, the Lagrangian is a scalar function $L: T Q \rightarrow \mathbb{R}$ which "determines everything". And the conservation of the generalized momentum $p_{n}$ conjugate to a cyclic coordinate $q_{n}, p_{n} \equiv p_{n}(q, \dot{q})=c_{n}$, means that the motion of the system is confined to a level set $p_{n}^{-1}\left(c_{n}\right)$ : where this level set is a $(2 n-1)$-dimensional sub-manifold of $T Q$.

\footnotetext{
${ }^{6} \mathrm{~A}$ note for afficionados. Of the three main pillars of elementary differential geometry - the implicit function theorem, the local existence and uniqueness of solutions of ordinary differential equations, and Frobenius' theorem - this paper will use the first only implicitly (!), and the second explicitly in Sections 3 and 4 . The third will not be used.
} 
But I must admit at the outset that working with $T Q$ involves limiting our discussion to (a) time-independent Lagrangians and (b) time-independent coordinate transformations.

(a): Recall Section 2.1's assumptions that secured eq. 2.1. Velocity-dependent potentials and-or rheonomous constraints would prompt one to use what is often called the 'extended configuration space' $Q \times \mathbb{R}$, and-or the 'extended velocity phase space' $T Q \times \mathbb{R}$.

(b): So would time-dependent coordinate transformations. This is a considerable limitation from a philosophical viewpoint, since it excludes boosts, which are central to the philosophical discussion of spacetime symmetry groups, and especially of relativity principles. To give the simplest example: the Lagrangian of a free particle is just its kinetic energy, which can be made zero by transforming to the particle's rest frame; i.e. it is not invariant under boosts.

\subsubsection{The tangent bundle}

With these limitations admitted, we now describe Lagrangian mechanics on $T Q$, in five extended comments.

(1): $2 n$ first-order equations; the Hessian again:-

The Lagrangian equations of motion are now $2 n$ first-order equations for the functions $q^{i}(t), \dot{q}^{i}(t)$, falling in to two groups:

(a) the $n$ equations eq. 2.2, with the $\ddot{q}^{i}$ taken as the time derivatives of $\dot{q}^{i}$ with respect to $t$; i.e. we envisage using the Hessian condition eq. 2.3 to solve eq. 2.2 for the $\ddot{q}^{i}$, hard though this usually is to do in practice;

(b) the $n$ equations $\dot{q}^{i}=\frac{d q^{i}}{d t}$.

(2): Vector fields and solutions:-

(a): These $2 n$ first-order equations are equivalent to a vector field on $T Q$ : the 'dynamical vector field', or for short the 'dynamics'. I write it as $D$ (to distinguish it from the generic vector field $X, Y, \ldots)$.

(b): In the natural coordinates $\left(q^{i}, \dot{q}^{i}\right)$, the vector field $D$ is expressed as

$$
D=\dot{q}^{i} \frac{\partial}{\partial q^{i}}+\ddot{q}^{i} \frac{\partial}{\partial \dot{q}^{i}}
$$

and the rate of change of any dynamical variable $f$, taken as a scalar function on $T Q$, $f(q, \dot{q}) \in \mathbb{R}$ is given by

$$
\frac{d f}{d t}=\dot{q}^{i} \frac{\partial f}{\partial q^{i}}+\ddot{q}^{i} \frac{\partial f}{\partial \dot{q}^{i}}=D(f) .
$$

(c): So the Lagrangian $L$ determines the dynamical vector field $D$, and so (for given initial $q, \dot{q}$ ) a (locally unique) solution: an integral curve of $D, 2 n$ functions of time $q(t), \dot{q}(t)$ (with the first $n$ functions determining the latter). This separation of solutions/trajectories within $T Q$ is important for the visual and qualitative understanding of solutions. 
(3): Canonical momenta are 1-forms:-

Any point transformation, or any coordinate transformation $\left(q^{i}\right) \rightarrow\left(q^{i}\right)$, in the configuration manifold $Q$, induces a basis-change in the tangent space $T_{q}$ at $q \in Q$. Consider any vector $\tau \in T_{q}$ with components $\dot{q}^{i}$ in coordinate system $\left(q^{i}\right)$ on $Q$, i.e. $\tau=\frac{d}{d t}=\dot{q}^{i} \frac{\partial}{\partial q^{i}}$; (think of a motion through configuration $q$ with generalized velocity $\tau$ ). Its components $\dot{q}^{i}$ in the coordinate system $\left(q^{i}\right)$ (i.e. $\left.\tau=\dot{q}^{\prime} \frac{\partial}{\partial q^{\prime i}}\right)$ are given by applying the chain rule to $q^{i}=q^{\prime i}\left(q^{k}\right)$ :

$$
\dot{q}^{i} \equiv \frac{\partial q^{i}}{\partial q^{k}} \dot{q}^{k}
$$

so that we can "drop the dots":

$$
\frac{\partial \dot{q}^{i}}{\partial \dot{q}^{j}}=\frac{\partial q^{i}}{\partial q^{j}} .
$$

One easily checks, using eq. 2.11, that for any $L$, the canonical momenta $p_{i}:=\frac{\partial L}{\partial \dot{q}^{i}}$ form a 1 -form on $Q$, transforming under $\left(q^{i}\right) \rightarrow\left(q^{i}\right)$ by:

$$
p_{i}^{\prime}:=\frac{\partial L^{\prime}}{\partial \dot{q}^{\prime i}}=\frac{\partial q^{k}}{\partial q^{\prime i}} \frac{\partial L}{\partial \dot{q}^{k}} \equiv \frac{\partial q^{k}}{\partial q^{\prime i}} p_{k}
$$

That is, the canonical momenta defined by $L$ form a 1 -form field on $Q$. (We will later describe this as a cross-section of the cotangent bundle.)

(4): Geometric formulation of Lagrange's equations:-

We can formulate Lagrange's equations in a coordinate-independent way, by using three ingredients, namely:

(i): $L$ itself (a scalar, so coordinate-independent);

(ii): the vector field $D$ that $L$ defines; and

(iii): the 1-form on $T Q$ defined locally, in terms of the natural coordinates $\left(q^{i}, \dot{q}^{i}\right)$, by

$$
\theta_{L}:=\frac{\partial L}{\partial \dot{q}^{i}} d q^{i}
$$

(So the coefficients of $\theta_{L}$ for the other $n$ elements of the dual basis, the $d \dot{q}^{i}$ are defined to be zero.) This 1-form is called the canonical 1-form. We shall see that it plays a role in Noether's theorem, and is centre-stage in Hamiltonian mechanics.

We combine these three ingredients using the idea of the Lie derivative of a 1-form along a vector field.

We will write the Lie derivative of $\theta_{L}$ along the vector field $D$ on $T Q$, as $\mathcal{L}_{D} \theta_{L}$. (It is sometimes written as $L$; but we need the symbol $L$ for the Lagrangian-and later on, for left translation.) By the Leibniz rule, $\mathcal{L}_{D} \theta_{L}$ is:

$$
\mathcal{L}_{D} \theta_{L}=\left(\mathcal{L}_{D} \frac{\partial L}{\partial \dot{q}^{i}}\right) d q^{i}+\frac{\partial L}{\partial \dot{q}^{i}} \mathcal{L}_{D}\left(d q^{i}\right)
$$

But the Lie derivative of any scalar function $f: T Q \rightarrow \mathbb{R}$ along any vector field $X$ is just $X(f)$; and for the dynamical vector field $D$, this is just $\dot{f}=\frac{\partial f}{\partial q^{i}} \dot{q}^{i}+\frac{\partial f}{\partial \dot{q}^{i}} \ddot{q}^{i}$. So we 
have

$$
\mathcal{L}_{D} \theta_{L}=\left(\frac{d}{d t} \frac{\partial L}{\partial \dot{q}^{i}}\right) d q^{i}+\frac{\partial L}{\partial \dot{q}^{i}} d \dot{q}^{i}
$$

Rewriting the first term by the Lagrange equations, we get

$$
\mathcal{L}_{D} \theta_{L}=\left(\frac{\partial L}{\partial q^{i}}\right) d q^{i}+\frac{\partial L}{\partial \dot{q}^{i}} d \dot{q}^{i} \equiv d L
$$

We can conversely deduce the familiar Lagrange equations from eq. 2.16, by taking coordinates. So we conclude that these equations' coordinate-independent form is:

$$
\mathcal{L}_{D} \theta_{L}=d L
$$

(5): Towards the Hamiltonian framework:-

Finally, a comment about the Lagrangian framework's limitations as regards solving problems, and how they prompt the transition to Hamiltonian mechanics.

Recall the remark at the end of Section 2.1, that the $n$ equations eq. 2.2 are in general hard to solve for the $\ddot{q}^{i}\left(t_{0}\right)$ : they lie buried in the left hand side of eq. 2.2. On the other hand, the $n$ equations $\dot{q}^{i}=\frac{d q^{i}}{d t}$ (the second group of $n$ equations in (1) above) are as simple as can be.

This makes it natural to seek another $2 n$-dimensional space of variables, $\xi^{\alpha}$ say $(\alpha=1, \ldots, 2 n)$, in which:

(i): a motion is described by first-order equations, so that we have the same advantage as in $T Q$ that a unique trajectory passes through each point of the space; but in which

(ii): all $2 n$ equations have the simple form $\frac{d \xi^{\alpha}}{d t}=f_{\alpha}\left(\xi^{1}, \ldots \xi^{2 n}\right)$ for some set of functions $f_{\alpha}(\alpha=1, \ldots, 2 n)$.

Indeed, Hamiltonian mechanics provides exactly such a space: it is usually the cotangent bundle of the configuration manifold, instead of its tangent bundle. But before turning to that, we expound Noether's theorem in the current Lagrangian framework.

\section{Noether's theorem in Lagrangian mechanics}

\subsection{Preamble: a modest plan}

Any discussion of symmetry in Lagrangian mechanics must include a treatment of "Noether's theorem". The scare quotes are to indicate that there is more than one Noether's theorem. Quite apart from Noether's work in other branches of mathematics, her paper (1918) on symmetries and conserved quantities in Lagrangian theories has several theorems. I will be concerned only with applying her first theorem to finitedimensional systems. In short: it provides, for any continuous symmetry of a system's Lagrangian, a conserved quantity called the 'momentum conjugate to the symmetry'. 
I stress at the outset that the great majority of subsequent applications and commentaries (also for her other theorems, besides her first) are concerned with versions of the theorems for infinite (i.e. continuous) systems. In fact, the context of Noether's investigation was contemporary debate about how to understand conservation principles and symmetries in the "ultimate classical continuous system", viz. gravitating matter as described by Einstein's general relativity. This theory can be given a Lagrangian formulation: that is, the equations of motion, i.e. Einstein's field equations, can be deduced from a Hamilton's Principle with an appropriate Lagrangian. The contemporary debate was especially about the conservation of energy and the principle of general covariance (also known as: diffeomorphism invariance). General covariance prompts one to consider how a variational principle transforms under spacetime coordinate transformations that are arbitrary, in the sense of varying from point to point. This leads to the idea of "local" symmetries, which since Noether's time has been immensely fruitful in both classical and quantum physics, and in both a Lagrangian and Hamiltonian framework. ${ }^{7}$

So I agree that from the perspective of Noether's work, and its enormous later development, this Section's application of the first theorem to finite-dimensional systems is, as they say, "trivial". Furthermore, this application is easily understood, without having to adopt that perspective, or even having to consider infinite systems. In other words: its statement and proof are natural, and simple, enough that the nineteenth century masters of mechanics, like Hamilton, Jacobi and Poincaré, would certainly recognize it in their own work - allowing of course for adjustments to modern language. In fact, versions of it for the Galilei group of Newtonian mechanics and the Lorentz group of special relativity were published a few years before Noether's paper; (Brading and Brown (2003, p. 90); for details, cf. Kastrup (1987)). ${ }^{8}$

Nevertheless, it is worth expounding the finite-system version of Noether's first theorem. For:

(i): It generalizes Section 2.1's result about cyclic coordinates, and thereby the elementary theorems of the conservation of momentum, angular momentum and energy which that result encompasses. The main generalization is that the theorem does not assume we have identified a cyclic coordinate. But on the other hand: every symmetry in the Noether sense will arise from a cyclic coordinate in some system $q$ of generalized coordinates. (As we will see, this follows from the local existence and uniqueness of solutions of ordinary differential equations.)

(ii): This exposition will also prepare the way for our discussion of symmetry and

\footnotetext{
${ }^{7}$ Cf. Brading and Castellani (2003). Apart from papers specifically about Noether's theorem, this anthology's papers by Wallace, Belot and Earman (all 2003) are closest to this paper's concerns.

${ }^{8}$ Here again, 'versions of it' needs scare-quotes. For in what follows, I shall be more limited than these proofs, in two ways. (1): I limit myself, as I did in Section 2.2.1, both to time-independent Lagrangians and to time-independent transformations: so my discussion does not encompass boosts. (2): I will take a symmetry of $L$ to require that $L$ be the very same; whereas some treatments allow the addition to $L$ of the time-derivative of a function $G(q)$ of the coordinates $q$ - since such a time-derivative makes no difference to the Lagrange equations.
} 
conserved quantities in Hamiltonian mechanics. ${ }^{9}$

In this exposition, I will also discuss en passant the distinction between:

(i) the notion of symmetry at work in Noether's theorem, i.e. a symmetry of $L$, often called a variational symmetry; and

(ii) the notion of a symmetry of the set of solutions of a differential equation: often called a dynamical symmetry. This notion applies to all sorts of differential equations, and systems of them; not just to those with the form of Lagrange's equations (i.e. derivable from an variational principle). In short, this sort of symmetry is a map that sends any solution of the given equation(s) (in effect: a dynamically possible history of the system - a curve in the state-space) to some other solution. Finding such symmetries, and groups of them, is a central part of the modern theory of integration of differential equations (both ordinary and partial).

Broadly speaking, this notion is more general than that of a symmetry of $L$. Not only does it apply to many other sorts of differential equation. Also, for Lagrange's equations: a symmetry of $L$ is (with one caveat) a symmetry of the solutions, i.e. a dynamical symmetry - but the converse is false. ${ }^{10}$

In this Section, the plan is as follows. We define:

(i): a (continuous) symmetry as a vector field (on the configuration manifold $Q$ ) that generates a family of transformations under which the Lagrangian is invariant; (Section 3.2);

(ii): the momentum conjugate to a vector field, as (roughly) the rate of change of the Lagrangian with respect to the $\dot{q}$ s in the direction of the vector field; (Section 3.3).

These two definitions lead directly to Noether's theorem (Section 3.4): after all the stage-setting, the proof will be a one-liner application of Lagrange's equations.

\subsection{Vector fields and symmetries - variational and dynamical}

I need to expound three topics:

(1): the idea of a vector field on the configuration manifold $Q$; and how to lift it to $T Q$

(2): the definition of a variational symmetry;

(3): the contrast between (2) and the idea of dynamical symmetry.

Note that, as in previous Sections, I will often speak, for simplicity, "globally, not locally", i.e. as if the relevant scalar functions, vector fields etc. are defined on all of

\footnotetext{
${ }^{9}$ Other expositions of Noether's theorem for finite-dimensional Lagrangian mechanics include: Arnold (1989: 88-89), Desloge (1982: 581-586), Lanczos (1986: 401-405: emphasizing the variational perspective) and Johns (2005: Chapter 13). Butterfield (2004a, Section 4.7) is a more detailed version of this Section. Beware: though many textbooks of Hamiltonian mechanics cover the Hamiltonian version of Noether's theorem (which, as we will see, is stronger), they often do not label it as such; and if they do label it, they often do not relate it clearly to the Lagrangian version.

${ }^{10}$ An excellent account of this modern integration theory, covering both ordinary and partial differential equations, is given by Olver (2000). He also covers the Lagrangian case (Chapter 5 onwards), and gives many historical details especially about Lie's pioneering contributions.
} 
$Q$ or $T Q$. Of course, they need not be.

\subsubsection{Vector fields on $T Q$; lifting fields from $Q$ to $T Q$}

We recall first that a differentiable vector field on $Q$ is represented in a coordinate system $q=\left(q^{1}, \ldots, q^{n}\right)$ by $n$ first-order ordinary differential equations

$$
\frac{d q^{i}}{d \epsilon}=f^{i}\left(q^{1}, \ldots, q^{n}\right)
$$

A vector field generates a one-parameter family of active transformations: viz. passage along the vector field's integral curves, by a varying parameter-difference $\epsilon$. The vector field is called the infinitesimal generator of the family. It is common to write the parameter as $\tau$, but in this Section we use $\epsilon$ to avoid confusion with $t$, which often represents the time.

Similarly, a vector field defined on $T Q$ corresponds to a system of $2 n$ ordinary differential equations, and generates an active transformation of $T Q$. But I will consider only vector fields on $T Q$ that mesh with the structure of $T Q$ as a tangent bundle, in the sense that they are induced by vector fields on $Q$, in the following natural way.

This induction has two ingredient ideas.

First, any curve in $Q$ (representing a possible state of motion) defines a corresponding curve in $T Q$, because the functions $q^{i}(t)$ define the functions $\dot{q}^{i}(t)$. (Here $t$ is the parameter of the curve.) More formally: given any curve in configuration space, $\phi: I \subset$ $\mathbb{R} \rightarrow Q$, with coordinate expression in the $q$-system $t \in I \mapsto q(\phi(t)) \equiv q(t)=q^{i}(t)$, we define its extension to $T Q$ to be the curve $\Phi: I \subset \mathbb{R} \rightarrow T Q$ given in the corresponding coordinates by $q^{i}(t), \dot{q}^{i}(t)$.

Second, any vector field $X$ on $Q$ generates displacements in any possible state of motion, represented by a curve in $Q$ with coordinate expression $q^{i}=q^{i}(t)$. Namely: for a given value of the parameter $\epsilon$, the displaced state of motion is represented by the curve in $\mathrm{Q}$

$$
q^{i}(t)+\epsilon X^{i}\left(q^{i}(t)\right)
$$

Putting these ingredients together: we first displace a curve within $Q$, and then extend the result to $T Q$. Namely, the extension to $T Q$ of the (curve representing) the displaced state of motion is given by the $2 n$ functions, in two groups each of $n$ functions, for the $(q, \dot{q})$ coordinate system

$$
q^{i}(t)+\epsilon X^{i}\left(q^{i}(t)\right) \text { and } \dot{q}^{i}(t)+\epsilon Y^{i}\left(q^{i}(t), \dot{q}^{i}\right)
$$

where $Y$ is defined to be the vector field on $T Q$ that is the derivative along the original state of motion of $X$. That is:

$$
Y^{i}(q, \dot{q}):=\frac{d X^{i}}{d t}=\Sigma_{j} \frac{\partial X^{i}}{\partial q^{j}} \dot{q}^{j}
$$


Thus displacements by a vector field within $Q$ are lifted to $T Q$. The vector field $X$ on $Q$ lifts to $T Q$ as $\left(X, \frac{d X}{d t}\right)$; i.e. it lifts to the vector field that sends a point $\left(q^{i}, \dot{q}^{i}\right) \in T Q$ to $\left(q^{i}+\epsilon X^{i}, \dot{q}^{i}+\epsilon \frac{d X^{i}}{d t}\right){ }^{11}$

\subsubsection{The definition of variational symmetry}

To define variational symmetry, I begin with the integral notion and then give the differential notion. The idea is that the Lagrangian $L$, a scalar $L: T Q \rightarrow \mathbb{R}$, should be invariant under all the elements of a one-parameter family of active transformations $\theta_{\epsilon}: \epsilon \in I \subset \mathbb{R}$ : at least in a neighbourhood of the identity map corresponding to $\epsilon=0$, $\theta_{0} \equiv i d_{U}$. (Here $U$ is some open subset of $T Q$, maybe not all of it.)

That is, we define the family $\theta_{\epsilon}: \epsilon \in I \subset \mathbb{R}$ to be a variational symmetry of $L$ if $L$ is invariant under the transformations: $L=L \circ \theta_{\epsilon}$, at least around $\epsilon=0$. (We could use the correspondence between active and passive transformations to recast this definition, and what follows, in terms of a passive notion of symmetry as sameness of $L$ 's functional form in different coordinate systems. I leave this as an exercise! Or cf. Butterfield (2004a: Section 4.7.2).)

For the differential notion of variational symmetry, we of course use the idea of a vector field. But we also impose Section 3.2.1's restriction to vector fields on $T Q$ that are induced by vector fields on $Q$. So we define a vector field $X$ on $Q$ that generates a family of active transformations $\theta_{\epsilon}$ on $T Q$ to be a variational symmetry of $L$ if the first derivative of $L$ with respect to $\epsilon$ is zero, at least around $\epsilon=0$. More precisely: writing

$$
L \circ \theta_{\epsilon}=L\left(q^{i}+\epsilon X^{i}, \dot{q}^{i}+\epsilon Y^{i}\right) \text { with } Y^{i}=\Sigma_{j} \frac{\partial X^{i}}{\partial q^{j}} \dot{q}^{j},
$$

we say $X$ is a variational symmetry iff the first derivative of $L$ with respect to $\epsilon$ is zero (at least around $\epsilon=0$ ). That is: $X$ is a variational symmetry iff

$$
\Sigma_{i} X^{i} \frac{\partial L}{\partial q^{i}}+\Sigma_{i} Y^{i} \frac{\partial L}{\partial \dot{q}^{i}}=0 \quad \text { with } Y^{i}=\Sigma_{j} \frac{\partial X^{i}}{\partial q^{j}} \dot{q}^{j}
$$

\subsubsection{A contrast with dynamical symmetries}

The general notion of a dynamical symmetry, i.e. a symmetry of some equations of motion (whether Euler-Lagrange or not), is not needed for Section 3.4's presentation of Noether's theorem. But the notion is so important that I must mention it, though only to contrast it with variational symmetries.

The general definition is roughly as follows. Given any system of differential equations, $\mathcal{E}$ say, a dynamical symmetry of the system is an active transformation $\zeta$ on the

\footnotetext{
${ }^{11}$ I have discussed this in terms of some system $(q, \dot{q})$ of coordinates. But the definitions of extensions and displacements are in fact coordinate-independent. Besides, one can show that the operations of displacing a curve within $Q$, and extending it to $T Q$, commute to first order in $\epsilon$ : the result is the same for either order of the operations.
} 
system $\mathcal{E}$ 's space of both independent variables, $x_{j}$ say, and dependent variables $y^{i}$ say, such that any solution of $\mathcal{E}, y^{i}=f^{i}\left(x_{j}\right)$ say, is carried to another solution. For a precise definition, cf. Olver (2000: Def. 2.23, p. 93), and his ensuing discussion of the induced action (called 'prolongation') of the transformation $\zeta$ on the spaces of (in general, partial) derivatives of the $y$ 's with respect to the $x$ s (i.e. jet spaces).

As I said in Section 3.1, groups of symmetries in this sense play a central role in the modern theory of differential equations: not just in finding new solutions, once given a solution, but also in integrating the equations. For some main theorems stating criteria (in terms of prolongations) for groups of symmetries, cf. Olver (2000: Theorem 2.27, p. 100, Theorem 2.36, p. 110, Theorem 2.71, p. 161).

But for present purposes, it is enough to state the rough idea of a one-parameter group of dynamical symmetries (without details about prolongations!) for Lagrange's equations in the familiar form, eq. 2.1.

In this simple case, there is just one independent variable $x:=t$, so that:

(a): we are considering ordinary, not partial, differential equations, with $n$ dependent variables $y^{i}:=q^{i}(t)$.

(b): prolongations correspond to lifts of maps on $Q$ to maps on $T Q$; cf. Section 3.2.1.

Furthermore, in line with the discussion following Lagrange's equations eq. 2.1, the time-independence of the Lagrangian (time being a cyclic coordinate) means we can define dynamical symmetries $\zeta$ in terms of active transformations on the tangent bundle, $\theta: T Q \rightarrow T Q$, that are lifted from active transformations on $Q$. In effect, we define such a map $\zeta$ by just adjoining to any such $\theta: T Q \rightarrow T Q$ the identity map on the time variable $i d: t \in \mathbb{R} \mapsto t$. (More formally: $\zeta:(q, \dot{q}, t) \in T Q \times \mathbb{R} \mapsto(\theta(q, \dot{q}), t) \in T Q \times \mathbb{R}$.)

Then we define in the usual way what it is for a one-parameter family of such maps $\zeta_{s}: s \in I \subset \mathbb{R}$ to be a (local) one-parameter group of dynamical symmetries (for Lagrange's equations eq. 2.1): namely, if any solution curve $q(t)$ (equivalently: its extension $q(t), \dot{q}(t)$ to $T Q$ ) of the Lagrange equations is carried by each $\zeta_{s}$ to another solution curve, with the $\zeta_{s}$ for different $s$ composing in the obvious way, for $s$ close enough to $0 \in I$.

And finally: we also define (in a manner corresponding to the discussion at the end of Section 3.2.2) a differential, as against integral, notion of dynamical symmetry. Namely, we say a vector field $X$ on $Q$ is a dynamical symmetry if its lift to $T Q$ (more precisely: its lift, with the identity map on the time variable adjoined) is the infinitesimal generator of such a one-parameter family $\zeta_{s}$.

For us, the important point is that this notion of a dynamical symmetry is different from Section 3.2.2's notion of a variational symmetry. ${ }^{12}$ As I announced in Section 3.1, a variational symmetry is (with one caveat) a dynamical symmetry-but the converse is false. Fortunately, the same simple example will serve both to show the subtlety

\footnotetext{
${ }^{12}$ Since the Lagrangian $L$ is especially associated with variational principles, while the dynamics is given by equations of motion, calling Section 3.2.2's notion 'variational symmetry', and this notion 'dynamical symmetry' is a good and widespread usage. But beware: it is not universal.
} 
about the first implication, and as a counterexample to the converse implication. This example is the two-dimensional harmonic oscillator. ${ }^{13}$

The usual Lagrangian is, with cartesian coordinates written as $q \mathrm{~s}$, and the contravariant indices written for clarity as subscripts:

$$
L_{1}=\frac{1}{2}\left[\dot{q}_{1}^{2}+\dot{q}_{2}^{2}-\omega^{2}\left(q_{1}^{2}+q_{2}^{2}\right)\right] ;
$$

giving as Lagrange equations:

$$
\ddot{q}_{i}+\omega^{2} q_{i}=0, \quad i=1,2 .
$$

But these Lagrange equations, i.e. the same dynamics, are also given by

$$
L_{2}=\dot{q}_{1} \dot{q}_{2}-\omega^{2} q_{1} q_{2}
$$

The rotations in the plane are of course a variational symmetry of $L_{1}$, and a dynamical symmetry of eq. 3.8. But they are not a variational symmetry of $L_{2}$. So a dynamical symmetry need not be a variational one. Besides, these equations contain another example to the same effect. Namely, the "squeeze" transformations

$$
q_{1}^{\prime}:=e^{\eta} q_{1}, q_{2}^{\prime}:=e^{-\eta} q_{2}
$$

are a dynamical symmetry of eq. 3.8 , but not a variational symmetry of $L_{1}$. So again: a dynamical symmetry need not be a variational one. ${ }^{14}$

I turn to the first implication: that every variational symmetry is a dynamical symmetry. This is true: general and abstract proofs (applying also to continuous systems i.e. field theories) can be found in Olver (2000: theorem 4.14, p. 255; theorem 4.34 , p. 278; theorem 5.53, p. 332).

But beware of a condition of the theorem. (This is the caveat mentioned at the end of Section 3.1.) The theorem requires that all the variables $q$ (for continuous systems: all the fields $\phi$ ) be subject to Hamilton's Principle. The need for this condition is shown by rotations in the plane, which are a variational symmetry of the familiar Lagrangian $L_{1}$ above. But it is easy to show that such a rotation is a dynamical symmetry of one of the Lagrange equations, say the equation for the variable $q_{1}$

$$
\ddot{q}_{1}+\omega^{2} q_{1}=0,
$$

only if the corresponding Lagrange equation holds for $q_{2}$.

\footnotetext{
${ }^{13}$ All the material to the end of this Subsection is drawn from Brown and Holland (2004a); cf. also their (2004). The present use of the harmonic oscillator example also occurs in Morandi et al (1990: 203-204).

${ }^{14}$ In the light of this, you might ask about a more restricted implication: viz. must every dynamical symmetry of a set of equations of motion be a variational symmetry of some or other Lagrangian that yields the given equations as the Euler-Lagrange equations of Hamilton's Principle? Again, the answer is No for the simple reason that there are many (sets of) equations of motion that are not Euler-Lagrange equations of any Lagrangian, and yet have dynamical symmetries.

Wigner (1954) gives an example. The general question of under what conditions is a set of ordinary differential equations the Euler-Lagrange equations of some Hamilton's Principle is the inverse problem of Lagrangian mechanics. It is a large subject with a long history; cf. e.g. Santilli (1979), Lopuszanski (1999).
} 


\subsection{The conjugate momentum of a vector field}

Now we define the momentum conjugate to a vector field $X$ to be the scalar function on $T Q$ :

$$
p_{X}: T Q \rightarrow \mathbb{R} ; p_{X}=\Sigma_{i} X^{i} \frac{\partial L}{\partial \dot{q}^{i}}
$$

(For a time-dependent Lagrangian, $p_{X}$ would be a scalar function on $T Q \times \mathbb{R}$, with $\mathbb{R}$ representing time.)

We shall see in the next Subsection's examples that this definition generalizes in an appropriate way Section 2.1's definition of the momentum conjugate to a coordinate $q$.

But first note that it is an improvement in the sense that, while the momentum conjugate to a coordinate $q$ depends on the choice made for the other coordinates, the momentum $p_{X}$ conjugate to a vector field $X$ is independent of the coordinates chosen. Though this point is not needed in order to prove Noether's theorem, here is the proof.

We first apply the chain-rule to $L=L\left(q^{\prime}(q), \dot{q}^{\prime}(q, \dot{q})\right)$ and eq. 2.11 ("cancellation of the dots"), to get

$$
\frac{\partial L}{\partial \dot{q}^{i}}=\Sigma_{j} \frac{\partial L}{\partial \dot{q}^{\prime j}} \frac{\partial \dot{q}^{\prime j}}{\partial \dot{q}^{i}}=\Sigma_{j} \frac{\partial L}{\partial \dot{q}^{\prime j}} \frac{\partial q^{\prime j}}{\partial q^{i}} .
$$

Then using the transformation law for components of a vector field

$$
X^{\prime i}=\Sigma_{j} \frac{\partial q^{i}}{\partial q^{j}} X^{j}
$$

and relabelling $i$ and $j$, we deduce:

$$
p_{X}^{\prime}=\Sigma_{i} X^{\prime i} \frac{\partial L}{\partial \dot{q}^{\prime i}}=\Sigma_{i j} X^{j} \frac{\partial q^{\prime i}}{\partial q^{j}} \frac{\partial L}{\partial \dot{q}^{\prime i}}=\Sigma_{i j} X^{i} \frac{\partial q^{\prime j}}{\partial q^{i}} \frac{\partial L}{\partial \dot{q}^{\prime j}}=\Sigma_{i} X^{i} \frac{\partial L}{\partial \dot{q}^{i}} \equiv p_{X}
$$

Finally, I remark incidentally that in the geometric formulation of Lagrangian mechanics (Section 2.2) , the coordinate-independence of $p_{X}$ becomes, unsurprisingly, a triviality. Namely: $p_{X}$ is obviously the contraction of $X$ as lifted to $T Q$ with the canonical 1-form on $T Q$ that we defined in eq. 2.13:

$$
\theta_{L}:=\frac{\partial L}{\partial \dot{q}^{i}} d q^{i}
$$

We will return to this at the end of Section 3.4.1.

\subsection{Noether's theorem; and examples}

Given just the definition of conjugate momentum, eq. 3.12, the proof of Noether's theorem is immediate. (The interpretation and properties of this momentum, discussed in the last Subsection, are not needed.) The theorem says: 
Noether's theorem for Lagrangian mechanics If $X$ is a (variational) symmetry of a system with Lagrangian $L(q, \dot{q}, t)$, then $X$ 's conjugate momentum is a constant of the motion.

Proof: We just calculate the derivative of the momentum eq. 3.12 along the solution curves in $T Q$, and apply Lagrange's equations and the definitions of $Y^{i}$, and of symmetry eq. 3.6:

$$
\begin{array}{r}
\frac{d p}{d t}=\Sigma_{i} \frac{d X^{i}}{d t} \frac{\partial L}{\partial \dot{q}^{i}}+\Sigma_{i} X^{i} \frac{d}{d t}\left(\frac{\partial L}{\partial \dot{q}^{i}}\right) \\
=\Sigma_{i} Y^{i} \frac{\partial L}{\partial \dot{q}^{i}}+\Sigma_{i} X^{i} \frac{\partial L}{\partial q^{i}}=0 . \text { QED. }
\end{array}
$$

Examples:- This proof, though neat, is a bit abstract! So here are two examples, both of which return us to examples we have already seen.

(1): The first example is a shift in a cyclic coordinate $q^{n}$ : i.e. the case with which our discussion of Noether's theorem began at the end of Section 2.1. So suppose $q^{n}$ is cyclic, and define a vector field $X$ by

$$
X^{1}=0, \ldots, X^{n-1}=0, X^{n}=1 .
$$

So the displacements generated by $X$ are translations by an amount $\epsilon$ in the $q^{n}$ direction. Then $Y^{i}:=\frac{d X^{i}}{d t}$ vanishes, and the definition of (variational) symmetry eq. 3.6 reduces to

$$
\frac{\partial L}{\partial q^{n}}=0
$$

So since $q^{n}$ is assumed to be cyclic, $X$ is a symmetry. And the momentum conjugate to $X$, which Noether's theorem tells us is a constant of the motion, is the familiar one:

$$
p_{X}:=\Sigma_{i} X^{i} \frac{\partial L}{\partial \dot{q}^{i}}=\frac{\partial L}{\partial \dot{q}^{n}}
$$

As mentioned in Section 3.1, this example is universal, in that every symmetry $X$ arises, around any point where $X$ is non-zero, from a cyclic coordinate in some local system of coordinates. This follows from the basic theorem about the local existence and uniqueness of solutions of ordinary differential equations. We can state the theorem as follows; (cf. e.g. Arnold (1973: 48-49, 77-78, 249-250), Olver (2000: Prop 1.29)).

Consider a system of $n$ first-order ordinary differential equations on an open subset $U$ of an $n$-dimensional manifold

$$
\dot{q}^{i}=X^{i}(q) \equiv X^{i}\left(q^{1}, \ldots, q^{n}\right), q \in U
$$

equivalently, a vector field $X$ on $U$. Let $q_{0}$ be a non-singular point of the vector field, i.e. $X\left(q_{0}\right) \neq 0$. Then in a sufficiently small neighbourhood $V$ of $q_{0}$, there is a coordinate 
system (formally, a diffeomorphism $f: V \rightarrow W \subset \mathbb{R}^{n}$ ) such that, writing $y_{i}: \mathbb{R}^{n} \rightarrow \mathbb{R}$ for the standard coordinates on $W$ and $\mathbf{e}_{i}$ for the $i$ th standard basic vector of $\mathbb{R}^{n}$, eq. 3.21 goes into the very simple form

$$
\dot{\mathbf{y}}=\mathbf{e}_{n} ; \text { i.e. } \dot{y}_{n}=1, \quad \dot{y}_{1}=\dot{y}_{2}=\ldots=\dot{y}_{n-1}=0 \text { in } W \text {. }
$$

(In terms of the tangent map (also known as: push-forward) $f_{*}$ on tangent vectors that is induced by $f: f_{*}(X)=\mathbf{e}_{n}$ in $W$.) On account of eq. 3.22's simple form, Arnold suggests the theorem might well be called the 'rectification theorem'.

We should note two points about the theorem:

(i): The rectifying coordinate system $f$ may of course be very hard to find. So the theorem by no means makes all problems "trivially soluble"; cf. again footnote 4.

(ii): The theorem has an immediate corollary about local constants of the motion. Namely: $n$ first-order ordinary differential equations have, locally, $n-1$ functionally independent constants of the motion (also known as: first integrals). They are given, in the above notation, by $y_{1}, \ldots, y_{n-1}$.

We now apply the rectification theorem, so as to reverse the reasoning in the above example of $q^{n}$ cyclic. That is: assuming $X$ is a symmetry, let us rectify it-i.e. let us pass to a coordinate system $(q)$ such that eq. 3.18 holds. Then, as above, $Y^{i}:=\frac{d X^{i}}{d t}$ vanishes; and $X$ 's being a (variational) symmetry, eq. 3.6, reduces to $q^{n}$ being cyclic; and the momentum conjugate to $X, p_{X}$ reduces to the familiar conjugate momentum $p_{n}=\frac{\partial L}{\partial \dot{q}^{n}}$. Thus every symmetry $X$ arises locally from a cyclic coordinate $q^{n}$ and the corresponding conserved momentum is $p_{n}$. (But note that this may hold only "very locally": the domain $V$ of the coordinate system $f$ in which $X$ generates displacements in the direction of the cyclic coordinate $q^{n}$ can be smaller than the set $U$ on which $X$ is a symmetry.)

In Section 5.3, the fact that every symmetry arises locally from a cyclic coordinate will be important for understanding the Hamiltonian version of Noether's theorem.

(2): Let us now look at our previous example, the angular momentum of a free particle (eq. 2.6), in the cartesian coordinate system, i.e. a coordinate system without cyclic coordinates. So let $q_{1}:=x, q_{2}:=y, q_{3}:=z$. (In this example, subscripts will again be a bit clearer.) Then a small rotation about the $x$-axis

$$
\delta x=0, \quad \delta y=-\epsilon z, \quad \delta z=\epsilon y
$$

corresponds to a vector field $X$ with components

$$
X_{1}=0, \quad X_{2}=-q_{3}, \quad X_{3}=q_{2}
$$

so that the $Y_{i}$ are

$$
Y_{1}=0, \quad Y_{2}=-\dot{q}_{3}, \quad X_{3}=\dot{q}_{2}
$$

For the Lagrangian

$$
L=\frac{1}{2} m\left(\dot{q}_{1}^{2}+\dot{q}_{2}^{2}+\dot{q}_{3}^{2}\right)
$$


$X$ is a (variational) symmetry since the definition of symmetry eq. 3.6 now reduces to

$$
\Sigma_{i} X_{i} \frac{\partial L}{\partial q_{i}}+\Sigma_{i} Y_{i} \frac{\partial L}{\partial \dot{q}_{i}}=-\dot{q}_{3} \frac{\partial L}{\partial \dot{q}_{2}}+\dot{q}_{2} \frac{\partial L}{\partial \dot{q}_{3}}=0
$$

So Noether's theorem then tells us that $X$ 's conjugate momentum is

$$
p_{X}:=\Sigma_{i} X_{i} \frac{\partial L}{\partial \dot{q}_{i}}=X_{2} \frac{\partial L}{\partial \dot{q}_{2}}+X_{3} \frac{\partial L}{\partial \dot{q}_{3}}=-m z \dot{y}+m y \dot{z}
$$

which is indeed the $x$-component of angular momentum.

\subsubsection{A geometrical formulation}

We can give a geometric formulation of Noether's theorem by using the vanishing of the Lie derivative to express constancy along the integral curves of a vector field. There are two vector fields on $T Q$ to consider: the dynamical vector field $D$ (cf. eq. 2.8), and the lift to $T Q$ of the vector field $X$ that is the variational symmetry.

I will now write $\bar{X}$ for this lift. So given the vector field $X$ on $Q$

$$
X=X^{i}(q) \frac{\partial}{\partial q^{i}}
$$

the lift $\bar{X}$ of $X$ to $T Q$ is, by eq. 3.4,

$$
\bar{X}=X^{i}(q) \frac{\partial}{\partial q^{i}}+\frac{\partial X^{i}(q)}{\partial q^{j}} \dot{q}^{j} \frac{\partial}{\partial \dot{q}^{i}}
$$

where the $q$ argument of $X^{i}$ emphasises that the $X^{i}$ do not depend on $\dot{q}$.

That $X$ is a variational symmetry means that in $T Q$, the Lie derivative of $L$ along the lift $\bar{X}$ vanishes: $\mathcal{L}_{\bar{X}} L=0$. On the other hand, we know from eq. 3.16 that the momentum $p_{X}$ conjugate to $X$ is the contraction $<;>$ of $\bar{X}$ with the canonical 1-form $\theta_{L}:=\frac{\partial L}{\partial \dot{q}^{i}} d q^{i}$ on $T Q:$

$$
p_{X}:=X^{i} \frac{\partial L}{\partial \dot{q}^{i}} \equiv<\bar{X} ; \theta_{L}>
$$

So Noether's theorem says:

$$
\text { If } \mathcal{L}_{\bar{X}} L=0 \text {, then } \mathcal{L}_{D}<\bar{X} ; \theta_{L}>=0 \text {. }
$$

Note finally that eq. 3.31 shows that the theorem has no converse. That is: given that a dynamical variable $p: T Q \rightarrow \mathbb{R}$ is a constant of the motion, $\mathcal{L}_{D} p=0$, there is no single vector field $\bar{X}$ on $T Q$ such that $p=\left\langle\bar{X} ; \theta_{L}>\right.$. For given such a $\bar{X}$, one could get another by adding any field $\bar{Y}$ for which $\left\langle\bar{Y} ; \theta_{L}\right\rangle=0$. However, we will see in Section 5.2 that in Hamiltonian mechanics a constant of the motion does determine a corresponding vector field on the state space. 


\section{Hamiltonian mechanics introduced}

\subsection{Preamble}

From now on this paper adopts the Hamiltonian framework. As we shall see, its description of symmetry and conserved quantities is in various ways more straightforward and powerful than that of the Lagrangian framework.

The main idea is to replace the $\dot{q}$ s by the canonical momenta, the $p$ s. More generally, the state-space is no longer the tangent bundle $T Q$ but a phase space $\Gamma$, which we take to be the cotangent bundle $T^{*} Q$. (Here, the phrase 'we take to be' just signals the fact that eventually, in Section 6.8, we will glimpse a more general kind of Hamiltonian state-space, viz. Poisson manifolds.)

Admittedly, the theory on $T Q$ given by Lagrange's equations eq. 2.1 is equivalent to the Hamiltonian theory on $T^{*} Q$ given by eq. 4.5 below, once we assume the Hessian condition eq. 2.3.

But of course, theories can be formally equivalent, but different as regards their power for solving problems, their heuristic value and even their interpretation. In our case, two advantages of Hamiltonian mechanics over Lagrangian mechanics are commonly emphasised. (i): The first concerns its greater power or flexibility for describing a given system, that Lagrangian methods can also describe (and so its greater power for solving problems about such a system). (ii): The second concerns the broader idea of describing other systems. In more detail:-

(i): Hamiltonian mechanics replaces the group of point transformations, $q \rightarrow q^{\prime}$ on $Q$, together with their lifts to $T Q$, by a "corresponding larger" group of transformations on $\Gamma$, the group of canonical transformations (also known as, for the standard case where $\Gamma=T^{*} Q$ : the symplectic group).

This group "corresponds" to the point transformations (and their lifts) in that while for any Lagrangian $L$, Lagrange's equations eq. 2.1 are covariant under all the point transformations, Hamilton's equations eq. 4.5 below are (for any Hamiltonian $H)$ covariant under all canonical transformations. And it is a "larger" group because:

(a) any point transformation together with its lift to $T Q$ is a canonical transformation: (more precisely: it naturally defines a canonical transformation on $T^{*} Q$ );

(b) not every canonical transformation is thus induced by a point transformation; for a canonical transformation can "mix" the $q \mathrm{~s}$ and $p \mathrm{~s}$ in a way that point transformations and their lifts cannot.

There is a rich and multi-faceted theory of canonical transformations, to which there are three main approaches - generating functions, integral invariants and symplectic geometry. I will adopt the symplectic approach, but not need many details about it. In particular, we will need only a few details about how the "larger" group of canonical transformations makes for a more powerful version of Noether's theorem.

(ii): The Hamiltonian framework connects analytical mechanics with other fields of physics, especially statistical mechanics and optics. The first connection goes via 
canonical transformations, especially using the integral invariants approach. The second connection goes via Hamilton-Jacobi theory; (for a philosopher's exposition, with an eye on quantum theory, cf. Butterfield (2004b: especially Sections 7-9)). ${ }^{15}$

With its theme of symmetry and conservation, this paper will illustrate (i), greater power in describing a given system, rather than (ii), describing other systems. As to (i), we will see two main ways in which the Hamiltonian framework is more powerful than the Lagrangian one. First, cyclic coordinates will "do more work for us" (Section 4.2). Second, the Hamiltonian version of Noether's theorem is both: more powerful, thanks to the use of the "larger" group of canonical transformations; and more easily proven, thanks to the use of Poisson brackets (Section 5).

So from now on, the broad plan is as follows. After Section 4.2's deduction of Hamilton's equations, Section 4.3 introduces symplectic structure, starting from the "naive" form of the symplectic matrix. Section 5 presents Poisson brackets, and the Hamiltonian version of Noether's theorem. Finally, Section 6 gives a geometric perspective, corresponding to Section 2.2's geometric perspective on the Lagrangian framework.

\subsection{Hamilton's equations}

\subsubsection{The equations introduced}

Recall the vision in (5) of Section 2.2.2: that we seek $2 n$ new variables, $\xi^{\alpha}$ say, $\alpha=$ $1, \ldots, 2 n$ in which Lagrange's equations take the simple form

$$
\frac{d \xi^{\alpha}}{d t}=f_{\alpha}\left(\xi^{1}, \ldots \xi^{2 n}\right)
$$

We can find the desired variables $\xi^{\alpha}$ by using the canonical momenta

$$
p_{i}:=\frac{\partial L}{\partial \dot{q}^{i}}=: L_{\dot{q}^{i}}
$$

to write the $2 n$ Lagrange equations as

$$
\frac{d p_{i}}{d t}=\frac{\partial L}{d q^{i}} \quad ; \quad \frac{d q^{i}}{d t}=\dot{q}^{i}
$$

These are of the desired simple form, except that the right hand sides need to be written as functions of $(q, p, t)$ rather than $(q, \dot{q}, t)$. (Here and in the next two paragraphs, we temporarily allow time-dependence, since the deduction is unaffected: the time variable is "carried along unaffected". In the terms of Section 2.1, this means allowing nonscleronomous constraints and a time-dependent work-function $U$.)

\footnotetext{
${ }^{15}$ Of course, some aspects of Hamiltonian mechanics illustrate both (i) and (ii). For example, Liouville's theorem on the preservation of phase space volume illustrates both (i)'s integral invariants approach to canonical transformations and (ii)'s connection to statistical mechanics.
} 
For the second group of $n$ equations, this is in principle straightforward, given our assumption of a non-zero Hessian, eq. 2.3. This implies that we can invert eq. 4.2 so as to get the $n \dot{q}^{i}$ as functions of $(q, p, t)$. We can then apply this to the first group of equations; i.e. we substitute $\dot{q}^{i}(q, p, t)$ wherever $\dot{q}^{i}$ appears in any right hand side $\frac{\partial L}{d q^{i}}$.

But we need to be careful: the partial derivative of $L(q, \dot{q}, t)$ with respect to $q^{i}$ is not the same as the partial derivative of $\hat{L}(q, p, t):=L(q, \dot{q}(q, p, t), t)$ with respect to $q^{i}$, since the first holds fixed the $\dot{q} \mathrm{~s}$, while the second holds fixed the $p \mathrm{~s}$. A comparison of these partial derivatives leads, with algebra, to the result that if we define the Hamiltonian function by

$$
H(q, p, t):=p_{i} \dot{q}^{i}(q, p, t)-\hat{L}(q, p, t)
$$

then the $2 n$ equations eq. 4.3 go over to Hamilton's equations

$$
\frac{d p_{i}}{d t}=-\frac{\partial H}{\partial q^{i}} \quad ; \quad \frac{d q^{i}}{d t}=\frac{\partial H}{\partial p_{i}}
$$

So we have cast our $2 n$ equations in the simple form, $\frac{d \xi^{\alpha}}{d t}=f_{\alpha}\left(\xi^{1}, \ldots \xi^{2 n}\right)$, requested in (5) of Section 2.2. More explicitly: defining

$$
\xi^{\alpha}=q^{\alpha}, \quad \alpha=1, \ldots, n \quad ; \quad \xi^{\alpha}=p_{\alpha-n}, \quad \alpha=n+1, \ldots, 2 n
$$

Hamilton's equations become

$$
\dot{\xi}^{\alpha}=\frac{\partial H}{\partial \xi^{\alpha+n}}, \quad \alpha=1, \ldots, n \quad ; \quad \dot{\xi}^{\alpha}=-\frac{\partial H}{\partial \xi^{\alpha-n}}, \quad \alpha=n+1, \ldots, 2 n .
$$

To sum up: a single function $H$ determines, through its partial derivatives, the evolution of all the $q \mathrm{~s}$ and $p \mathrm{~s}$ - and so, the evolution of the state of the system.

\subsubsection{Cyclic coordinates in the Hamiltonian framework}

Just from the form of Hamilton's equations, we can immediately see a result that is significant for our theme of how symmetries and conserved quantities reduce the number of variables involved in a problem. In short, we can see that with Hamilton's equations in hand, cyclic coordinates will "do more work for us" than they do in the Lagrangian framework.

More specifically, recall the basic Lagrangian result from the end of Section 2.1, that the generalized momentum $p_{n}:=\frac{\partial L}{\partial \dot{q}^{n}}$ is conserved if, indeed iff, its conjugate coordinate $q^{n}$ is cyclic, $\frac{\partial L}{\partial q^{n}}=0$. And recall from Section 3.4 that this result underpinned Noether's theorem in the precise sense of being "universal" for it. Corresponding results hold in the Hamiltonian framework-but are in certain ways more powerful.

Thus we first observe that the transformation "from the $\dot{q}$ s to the $p \mathrm{~s}$ ", i.e. the transition between Lagrangian and Hamiltonian frameworks, does not involve the dependence on the $q \mathrm{~s}$. More precisely: partially differentiating eq. 4.4 with respect to 
$q^{n}$, we obtain

$$
\left.\frac{\partial H}{\partial q^{n}} \equiv \frac{\partial H}{\partial q^{n}}\right|_{p ; q^{i}, i \neq n}=-\left.\frac{\partial L}{\partial q^{n}} \equiv \frac{\partial L}{\partial q^{n}}\right|_{\dot{q} ; q^{i}, i \neq n}
$$

(The other two terms are plus and minus $p_{i} \frac{\partial \dot{q}^{i}}{\partial q^{n}}$, and so cancel.) So a coordinate $q^{n}$ that is cyclic in the Lagrangian sense is also cyclic in the obvious Hamiltonian sense, viz. that $\frac{\partial H}{\partial q^{n}}=0$. But by Hamilton's equations, this is equivalent to $\dot{p}_{n}=0$. So we have the result corresponding to the Lagrangian one: $p_{n}$ is conserved iff $q_{n}$ is cyclic (in the Hamiltonian sense).

We will see in Section 5.3 that this result underpins the Hamiltonian version of Noether's theorem; just as the corresponding Lagrangian result underpinned the Lagrangian version of Noether's theorem (cf. discussion after eq. 3.20).

But we can already see that this result gives the Hamiltonian formalism an advantage over the Lagrangian. In the latter, the generalized velocity corresponding to a cyclic coordinate, $q_{n}$ will in general still occur in the Lagrangian. The Lagrangian will be $L\left(q_{1}, \ldots, q_{n-1}, \dot{q}_{1}, \ldots, \dot{q}_{n}, t\right)$, so that we still face a problem in $n$ variables.

But in the Hamiltonian formalism, $p_{n}$ will be a constant of the motion, $\alpha$ say, so that the Hamiltonian will be $H\left(q_{1}, \ldots, q_{n-1}, p_{1}, \ldots, p_{n-1}, \alpha, t\right)$. So we now face a problem in $n-1$ variables, $\alpha$ being simply determined by the initial conditions. That is: after solving the problem in $n-1$ variables, $q_{n}$ is determined just by quadrature: i.e. just by integrating (perhaps numerically) the equation

$$
\dot{q}_{n}=\frac{\partial H}{\partial \alpha}
$$

where, thanks to having solved the problem in $n-1$ variables, the right-hand side is now an explicit function of $t$.

This result is very simple. But it is an important illustration of the power of the Hamiltonian framework. Indeed, Arnold remarks (1989: 68) that 'almost all the solved problems in mechanics have been solved by means of' it!

No doubt his point is, at least in part, that this result underpins the Hamiltonian version of Noether's theorem. But I should add that the result also motivates the study of various notions related to the idea of cyclic coordinates, such as constants of the motion being in involution (i.e. having zero Poisson bracket with each other), and a system being completely integrable (in the sense of Liouville). These notions have played a large part in the way that Hamiltonian mechanics has developed, especially in its theory of canonical transformations. And they play a large part in the way Hamiltonian mechanics has solved countless problems. But as announced in Section 4.1, this paper will not go into these aspects of Hamiltonian mechanics, since they are not needed for our theme of symmetry and conservation; (for a philosophical discussion of these aspects, cf. Butterfield 2005). 


\subsubsection{The Legendre transformation and variational principles}

To end this Subsection, I note two aspects of this transition from Lagrange's equations to Hamilton's. For, although I shall not need details about them, they each lead to a rich theory:

(i): The transformation "from the $\dot{q}$ s to the $p \mathrm{~s}$ " is the Legendre transformation. It has a striking geometric interpretation. In the simplest case, it concerns the fact that one can describe a smooth convex real function $y=f(x), f^{\prime \prime}(x)>0$, not by the pairs of its arguments and values $(x, y)$, but by the pairs of its gradients at points $(x, y)$ and the intercepts of its tangent lines with the $y$-axis. Given the non-zero Hessian (eq. 2.3 ), one readily proves various results: e.g. that the geometric interpretation extends to higher dimensions, and that the transformation is self-inverse, i.e. its square is the identity. For details, cf. e.g.: Arnold (1989: Chapters 3.14, 9.45.C), Courant and Hilbert (1953: Chapter IV.9.3; 1962, Chapter I.6), José and Saletan (1998: 212-217), Lanczos (1986: Chapter VI.1-4). The Legendre transformation is also described using modern geometry's idea of a fibre derivative; as we will see briefly in Section 6.7.

(ii): The transition to Hamilton's equations has achieved more than we initially sought with our eq. 4.1. Namely: all the $f_{\alpha}$, all the right hand sides in Hamilton's equations, are up to a sign, partial derivatives of a single function $H$. In the Hamiltonian framework, it is precisely this feature that underpins the possibility of expressing the equations of motion by variational principles; (of course, the Lagrangian framework has a corresponding feature). But as I mentioned, this paper does not discuss variational principles; for details cf. e.g. Lanczos (1986: Chapter VI.4) and Butterfield (2004: especially Section 5.2).

To sum up this introduction to Hamilton's equations:- Even once we set aside (i) and (ii), these equations mark the beginning of a rich and multi-faceted theory. At the centre lies the $2 n$-dimensional phase space $\Gamma$ coordinatized by the $q \mathrm{~s}$ and $p \mathrm{~s}$ : or more precisely, as we shall see later, the cotangent bundle $T^{*} Q$. The structure of Hamiltonian mechanics is encoded in the structure of $\Gamma$, and thereby in the coordinate transformations on $\Gamma$ that preserve this structure, especially the form of Hamilton's equations: the canonical transformations. As I mentioned in Section 4.1, these transformations can be studied from three main perspectives: generating functions, integral invariants and symplectic structure-but I shall only need the last.

\subsection{Symplectic forms on vector spaces}

I shall introduce symplectic structure by giving Hamilton's equations a yet more symmetric appearance. This will lead to some elementary ideas about area in $\mathbb{R}^{m}$ and symplectic forms on vector spaces: ideas which will later be "made local" by taking the relevant copy of $\mathbb{R}^{m}$ to be the tangent space at a point of a manifold. (As usually formulated, Hamiltonian mechanics is especially concerned with the case $m=2 n$.) 


\subsubsection{Time-evolution from the gradient of $H$}

Writing $\mathbf{1}$ and $\mathbf{0}$ for the $n \times n$ identity and zero matrices respectively, we define the $2 n \times 2 n$ symplectic matrix $\omega$ by

$$
\omega:=\left(\begin{array}{cc}
0 & 1 \\
-1 & 0
\end{array}\right)
$$

$\omega$ is antisymmetric, and has the properties, writing for the transpose of a matrix, that

$$
\tilde{\omega}=-\omega=\omega^{-1} \quad \text { so that } \quad \omega^{2}=-1 \quad ; \quad \text { also } \quad \operatorname{det} \omega=1 .
$$

Using $\omega$, Hamilton's equations eq. 4.7 get the more symmetric form, in matrix notation

$$
\dot{\xi}=\omega \frac{\partial H}{\partial \xi} \text {. }
$$

In terms of components, writing $\omega^{\alpha \beta}$ for the matrix elements of $\omega$, and defining $\partial_{\alpha}:=$ $\partial / \partial \xi^{\alpha}$, eq. 4.7 become

$$
\dot{\xi}^{\alpha}=\omega^{\alpha \beta} \partial_{\beta} H .
$$

Eq. 4.12 and 4.13 show how $\omega$ forms, from the naive gradient (column vector) $\nabla H$ of $H$ on the phase space $\Gamma$ of $q \mathrm{~s}$ and $p \mathrm{~s}$, the vector field on $\Gamma$ that gives the system's evolution: the Hamiltonian vector field, often written $X_{H}$. At a point $z=(q, p) \in \Gamma$, eq. 4.12 can be written

$$
X_{H}(z)=\omega \nabla H(z) .
$$

The vector field $X_{H}$ is also written as $D$ (for 'dynamics'), on analogy with the Lagrangian framework's vector field $D$ of eq. 2.8 in Section 2.2.

In Section 6, we will see how this definition of a vector field from a gradient, i.e. a covector or 1-form field, arises from $\Gamma$ 's being a cotangent bundle. More precisely, we will see that any cotangent bundle has an intrinsic symplectic structure that provides, at each point of the base-manifold, a natural i.e. basis-independent isomorphism between the tangent space and the cotangent space. For the moment, we:

(i) note a geometric interpretation of $\omega$ in terms of area (Section 4.3.2); and then

(ii) generalize the above discussion of $\omega$ into the definition of a symplectic form for a fixed vector space (Section 4.3.3).

\subsubsection{Interpretation in terms of areas}

Let us begin with the simplest possible case: $\mathbb{R}^{2} \ni(q, p)$, representing the phase space of a particle constrained to one spatial dimension. Here, the $2 \times 2$ matrix

$$
\omega:=\left(\begin{array}{cc}
0 & 1 \\
-1 & 0
\end{array}\right)
$$


defines the antisymmetric bilinear form on $\mathbb{R}^{2}$ :

$$
A:\left(\left(q^{1}, p_{1}\right),\left(q^{2}, p_{2}\right)\right) \in \mathbb{R}^{2} \times \mathbb{R}^{2} \mapsto q^{1} p_{2}-q^{2} p_{1} \in \mathbb{R}
$$

since

$$
q^{1} p_{2}-q^{2} p_{1}=\left(\begin{array}{ll}
q^{1} & p_{1}
\end{array}\right)\left(\begin{array}{cc}
0 & 1 \\
-1 & 0
\end{array}\right)\left(\begin{array}{c}
q^{2} \\
p_{2}
\end{array}\right)=\operatorname{det}\left(\begin{array}{cc}
q^{1} & q^{2} \\
p_{1} & p_{2}
\end{array}\right)
$$

It is easy to prove that $A\left(\left(q^{1}, p_{1}\right),\left(q^{2}, p_{2}\right)\right) \equiv q^{1} p_{2}-q^{2} p_{1}$ is the signed area of the parallelogram spanned by $\left(q^{1}, p_{1}\right),\left(q^{2}, p_{2}\right)$, where the sign is positive (negative) if the shortest rotation from $\left(q^{1}, p_{1}\right)$ to $\left(q^{2}, p_{2}\right)$ is anti-clockwise (clockwise).

Similarly in $\mathbb{R}^{2 n}$ : the matrix $\omega$ of eq. 4.10 defines an antisymmetric bilinear form on $\mathbb{R}^{2 n}$ whose value on a pair $(q, p) \equiv\left(q^{1}, \ldots q^{n} ; p_{1}, \ldots, p_{n}\right),\left(q^{\prime}, p^{\prime}\right) \equiv\left(q^{\prime 1}, \ldots q^{\prime n} ; p_{1}^{\prime}, \ldots, p_{n}^{\prime}\right)$ is the sum of the signed areas of the $n$ parallelograms formed by the projections of the vectors $(q, p),\left(q^{\prime}, p^{\prime}\right)$ onto the $n$ pairs of coordinate planes labelled $1, \ldots, n$. That is to say, the value is:

$$
\sum_{i=1}^{n} q^{i} p_{i}^{\prime}-q^{i} p_{i}
$$

This induction of bilinear forms from antisymmetric matrices can be generalized: there is a one-to-one correspondence between forms and matrices. In more detail: there is a one-to-one correspondence between antisymmetric bilinear forms on $\mathbb{R}^{2}$ and antisymmetric $2 \times 2$ matrices. It is easy to check that any such form, $\omega$ say, is given, for any basis $v, w$ of $\mathbb{R}^{2}$, by the matrix $\left(\begin{array}{cc}0 & \omega(v, w) \\ -\omega(v, w) & 0\end{array}\right)$. Similarly for any integer $n$ : one easily shows that there is a one-to-one correspondence between antisymmetric bilinear forms on $\mathbb{R}^{n}$ and antisymmetric $n \times n$ matrices. (In Hamiltonian mechanics as usually formulated, we consider the case where $n$ is even and the matrix is non-singular, as in eq. 4.10. But when one generalizes to Poisson manifolds (cf. Section 6.8) one allows $n$ to be odd, and the matrix to be singular.)

This geometric interpretation of $\omega$ is important for two reasons.

(i): The first reason is that the idea of an antisymmetric bilinear form on a copy of $\mathbb{R}^{2 n}$ is the main part of the definition of a symplectic form, which is the central notion in the usual geometric formulation of Hamiltonian mechanics. More details in Section 4.3.3, for a fixed copy of $\mathbb{R}^{2 n}$; and in Section 6, where the form is defined on many copies of $\mathbb{R}^{2 n}$, each copy being the tangent space at a point in the cotangent bundle $T^{*} Q$.

(ii): The second reason is that the idea of (signed) area underpins the theory of forms (1-forms, 2-forms etc.): i.e. antisymmetric multilinear functions on products of copies of $\mathbb{R}^{n}$. And when these copies of $\mathbb{R}^{n}$ are copies of the tangent space at (one and the same) point in a manifold, these forms lead to the whole theory of integration on manifolds. One needs this theory in order to make rigorous sense of any integration on a manifold beyond the most elementary (i.e. line-integrals); so it is crucial for almost any mathematical or physical theory using manifolds. In particular, it is crucial for 
Hamiltonian mechanics. So no wonder the maestro says that 'Hamiltonian mechanics cannot be understood without differential forms' (Arnold 1989, p. 163).

However, it turns out that this paper will not need many details about forms and the theory of integration. This is essentially because we focus only on solving mechanical problems, and simplifying them by appeals to symmetry. This means we will focus on line-integrals: viz. integrating with respect to time the equations of motion; or equivalently, integrating the dynamical vector field on the state space. We have already seen this vector field as $X_{H}$ in eq. 4.14; and we will see it again, for example in terms of Poisson brackets (eq. 5.14), and in geometric terms (Section 6). But throughout, the main idea will be as suggested by eq. 4.14: the vector field is determined by the symplectic matrix, "at" each point in the manifold $\Gamma$, acting on the gradient of the Hamiltonian function $H$.

So in short: focussing on line-integrals enables us to side-step most of the theory of forms. ${ }^{16}$

\subsubsection{Bilinear forms and associated linear maps}

We now generalize from the symplectic matrix $\omega$ to a symplectic form; in five extended comments.

\section{(1): Preliminaries:-}

Let $V$ be a (real finite-dimensional) vector space, with basis $e_{1}, \ldots, e_{i}, \ldots e_{n}$. We write $V^{*}$ for the dual space, and $e^{1}, \ldots, e^{i}, \ldots e^{n}$ for the dual basis: $e^{i}\left(e_{j}\right):=\delta_{j}^{i}$.

We recall that the isomorphism $e_{i} \mapsto e^{i}$ is basis-dependent: for a different basis, the corresponding isomorphism would be a different map. Only with the provision of appropriate extra structure would this isomorphism be basis-independent.

For physicists, the most familiar example of such a structure is the spacetime metric $\mathrm{g}$ in relativity theory. In terms of components, this basis-independence shows up in the way that $\mathbf{g}$ and its inverse lower and raise indices. As we will see in a moment, the underlying mathematical point is that because $\mathbf{g}$ is a bilinear form on a vector space $V$, i.e. $\mathbf{g}: V \times V \rightarrow \mathbb{R}$, and is non-degenerate, any $v \in V$ defines, independently of any choice of basis, an element of $V^{*}$ : viz. the map $u \in V \mapsto \mathbf{g}(u, v)$. (In fact, $V$ is the tangent space at a spacetime point; but this physical interpretation is irrelevant to the mathematical argument.) We will also see that Hamiltonian mechanics has a non-degenerate bilinear form, viz. a symplectic form, that similarly gives a basisindependent isomorphism between a vector space and its dual. (Roughly speaking, this vector space will be the $2 n$-dimensional space of the $q \mathrm{~s}$ and $p \mathrm{~s}$.)

On the other hand: for any vector space $V$, the isomorphism between $V$ and $V^{* *}$ given by

$$
e_{i} \mapsto\left[e_{i}\right] \in V^{* *}: e^{j} \in V^{*} \mapsto e^{j}\left(e_{i}\right)=\delta_{i}^{j}
$$

\footnotetext{
${ }^{16}$ But forms are essential for understanding integration over surfaces of dimension two or more: which one needs for the integral invariants approach to Hamiltonian mechanics, and its deep connection with Stokes' theorem.
} 
is basis-independent, and so we identify $e_{i}$ with $\left[e_{i}\right]$, and $V$ with $V^{* *}$. We will write $<;>$ (also written $<,>$ ) for the natural pairing (in either order) of $V$ and $V^{*}$ : e.g. $<e_{i} ; e^{j}>=<e^{j} ; e_{i}>=\delta_{i}^{j}$.

A linear map $A: V \rightarrow W$ induces (basis-independently) a transpose (aka: dual), written $\tilde{A}$ (or $A^{T}$ or $A^{*}$ ), $\tilde{A}: W^{*} \rightarrow V^{*}$ by

$$
\forall \alpha \in W^{*}, \forall v \in V: \tilde{A}(\alpha)(v) \equiv<\tilde{A}(\alpha) ; v>:=\alpha(A(v)) \equiv(\alpha \circ A)(v) .
$$

If $A: V \rightarrow W$ is a linear map between real finite-dimensional vector spaces, its matrix with respect to bases $e_{1}, \ldots, e_{i}, \ldots e_{n}$ and $f_{1}, \ldots, f_{j}, \ldots f_{m}$ of $V$ and $W$ is given by:

$$
A\left(e_{i}\right)=A_{i}^{j} f_{j} ; \quad \text { i.e. with } v=v^{i} e_{i}, \quad(A(v))^{j}=A_{i}^{j} v^{i} .
$$

So the upper index labels rows, and the lower index labels columns. Similarly, if $A: V \times W \rightarrow \mathbb{R}$ is a bilinear form, its matrix for these bases is defined as

$$
A_{i j}:=A\left(e_{i}, f_{j}\right)
$$

so that on vectors $v=v^{i} e_{i}, w=w^{j} f_{j}$, we have: $A(v, w)=v^{i} A_{i j} w^{j}$.

(2): Associated maps and forms:-

Given a bilinear form $A: V \times W \rightarrow \mathbb{R}$, we define the associated linear map $A^{b}: V \rightarrow W^{*}$ by

$$
A^{b}(v)(w):=A(v, w) .
$$

Then $A^{b}\left(e_{i}\right)=A_{i j} f^{j}$ : for both sides send any $w=w^{j} f_{j}$ to $A_{i j} w^{j}$. That is: the matrix of $A^{b}$ in the bases $e_{i}, f^{j}$ of $V$ and $W^{*}$ is $A_{i j}$ :

$$
\left[A^{b}\right]_{i j}=A_{i j}
$$

On the other hand, we can proceed from linear maps to associated bilinear forms. Given a linear map $B: V \rightarrow W^{*}$, we define the associated bilinear form $B^{\sharp}$ on $V \times$ $W^{* *} \cong V \times W$ by

$$
B^{\sharp}(v, w)=\langle B(v) ; w>.
$$

If we put $A^{\mathrm{b}}$ for $B$ in eq. 4.25, its associated bilinear form, acting on vectors $v=$ $v^{i} e_{i}, w=w^{j} f_{j}$, yields, by eq. 4.23:

$$
\left(A^{b}\right)^{\sharp}(v, w)=\left\langle A^{b}(v) ; w>=A(v, w) .\right.
$$

One similarly shows that if $B: V \rightarrow W^{*}$, then $\forall w \in W$ :

$$
\left(B^{\sharp}\right)^{b}(v)(w) \equiv<\left(B^{\sharp}\right)^{b}(v) ; w>=B(v)(w) \equiv<B(v) ; w>\text { so that }\left(B^{\sharp}\right)^{b}=B .
$$

So the flat and sharp operations, ${ }^{b}$ and ${ }^{\sharp}$, are inverses.

(3): Tensor products:-

It will sometimes be helpful to put the above ideas in terms of tensor products. If 
$v \in V, w \in W$, we can think of $v$ and $w$ as elements of $V^{* *}, W^{* *}$ respectively. So we define their tensor product as a bilinear form on $V^{*} \times W^{*}$ by requiring for all $\alpha \in V^{*}, \beta \in W^{*}$ :

$$
(v \otimes w)(\alpha, \beta):=v(\alpha) w(\beta) \equiv<v ; \alpha><w ; \beta>.
$$

Similarly for other choices of vector spaces or their duals. Given $\alpha \in V^{*}, \beta \in W^{*}$, their tensor product is a bilinear form on $V \times W$ :

$$
(\alpha \otimes \beta)(v, w):=\alpha(v) \beta(w) \equiv<v ; \alpha><w ; \beta>
$$

Similarly, we can think of $\alpha \in V^{*}, w \in W$ as elements of $V^{*}$ and $W^{* *}$ respectively, and so define their tensor product as a bilinear form on $V \times W^{*}$ :

$$
(\alpha \otimes w)(v, \beta):=\alpha(v) w(\beta) \equiv<v ; \alpha><w ; \beta>
$$

In this way we can express the linear map $A: V \rightarrow W$ in terms of tensor products. Since

$$
A\left(e_{i}\right)=A_{i}^{j} f_{j} \quad \text { iff } \quad<A\left(e_{i}\right) ; f^{j}>=A_{i}^{j}
$$

eq. 4.30 implies that

$$
A=A_{i}^{j} e^{i} \otimes f_{j} .
$$

Similarly, a bilinear form $A: V \times W \rightarrow \mathbb{R}$ with matrix $A_{i j}:=A\left(e_{i}, f_{j}\right)$ (cf. eq. 4.22) is:

$$
A=A_{i j} e^{i} \otimes f^{j}
$$

The definitions of tensor product eq. 4.28, 4.29 and 4.30 generalize to higher-rank tensors (i.e. multilinear maps whose domains have more than two factors). But we will not need these generalizations.

(4): Antisymmetric and non-degenerate forms:-

We now specialize to the forms and maps of central interest in Hamiltonian mechanics. We take $W=V, \operatorname{dim}(V)=n$, and define a bilinear form $\omega: V \times V \rightarrow \mathbb{R}$ to be:

(i): antisymmetric iff: $\omega\left(v, v^{\prime}\right)=-\omega\left(v, v^{\prime}\right)$;

(ii): non-degenerate iff: if $\omega\left(v, v^{\prime}\right)=0 \forall v^{\prime} \in V$, then $v=0$.

The form $\omega$ and its associated linear map $\omega^{b}: V \rightarrow V^{*}$ now have a square matrix $\omega_{i j}$ (cf. eq. 4.24). We define the rank of $\omega$ to be the rank of this matrix: equivalently, the dimension of the range $\omega^{b}(V)$.

We will also need the antisymmetrized version of eq. 4.29 that is definable when $W=V$. Namely, we define the wedge-product of $\alpha, \beta \in V^{*}$ to be the antisymmetric bilinear form on $V$, given by

$$
\alpha \wedge \beta:(v, w) \in V \times V \mapsto(\alpha(v))(\beta(w))-(\alpha(w))(\beta(v)) \in \mathbb{R} .
$$

(The connection with Section 4.3.2, especially eq. 4.18, will become clear in a moment; and will be developed in Section 6.2.A.) 
It is easy to show that for any bilinear form $\omega: V \times V \rightarrow \mathbb{R}: \omega$ is non-degenerate iff the matrix $\omega_{i j}$ is non-singular iff $\omega^{b}: V \rightarrow V^{*}$ is an isomorphism.

So a non-degenerate bilinear form establishes a basis-independent isomorphism between $V$ and $V^{*}$; cf. the discussion of the spacetime metric $\mathbf{g}$ in (1) at the start of this Subsection.

Besides, this isomorphism $\omega^{b}$ has an inverse, suggesting another use of the sharp notation, viz. $\omega^{\sharp}$ is defined to be $\left(\omega^{b}\right)^{-1}: V^{*} \rightarrow V$. The isomorphism $\omega^{\sharp}: V^{*} \rightarrow V$ corresponds to $\omega$ 's role, emphasised in Section 4.3.1, of defining a vector field $X_{H}$ from $d H$. (But we will see in a moment that the space $V$ implicitly considered in Section 4.3.1 had more structure than being just any finite-dimensional real vector space: viz. it was of the form $W \times W^{*}$.)

NB: This definition of $\sharp$ is of course not equivalent to our previous definition, in eq. 4.25, since:

(i): on our previous definition, ${ }^{\sharp}$ carried a linear map to a bilinear form, which reversed the passage by ${ }^{b}$ from bilinear form to linear map, in the sense that for a bilinear form $\omega$, we had $\left(\omega^{b}\right)^{\sharp}=\omega$; cf. eq. 4.26 ;

(ii): on the present definition, ${ }^{\sharp}$ carries a bilinear form $\omega: V \times V \rightarrow \mathbb{R}$ to a linear map $\omega^{\sharp}: V^{*} \rightarrow V$, which inverts ${ }^{b}$ in the sense (different from (i)) that

$$
\omega^{\sharp} \circ \omega^{b}=i d_{V} \text { and } \omega^{b} \circ \omega^{\sharp}=i d_{V^{*}} \text {. }
$$

So beware: though not equivalent, both definitions are used! But it is a natural ambiguity, in so far as the definitions "mesh". For example, one easily shows that our second definition, i.e. eq. 4.35 , is equivalent to a natural expression:

$$
\forall \alpha, \beta \in V^{*}:\left\langle\omega^{\sharp}(\alpha), \beta>:=\omega\left(\left(\omega^{b}\right)^{-1}(\alpha),\left(\omega^{b}\right)^{-1}(\beta)\right) .\right.
$$

It is also straightforward to show that for any bilinear form $\omega: V \times V \rightarrow \mathbb{R}$ : if $\omega$ is antisymmetric of rank $r \leq n \equiv \operatorname{dim}(V)$, then $r$ is even. That is: $r=2 s$ for some integer $s$, and there is a basis $e_{1}, \ldots, e_{i}, \ldots, e_{n}$ of $V$ for which $\omega$ has a simple expansion as wedge-products

$$
\omega=\Sigma_{i=1}^{s} e^{i} \wedge e^{i+s}
$$

equivalently, $\omega$ has the $n \times n$ matrix

$$
\omega=\left(\begin{array}{ccc}
0 & 1 & 0 \\
-1 & 0 & 0 \\
0 & 0 & 0
\end{array}\right)
$$

where 1 is the $s \times s$ identity matrix, and similarly for the zero matrices of various sizes. This normal form of antisymmetric bilinear forms is an analogue of the Gram-Schmidt theorem that an inner product space has an orthonormal basis, and is proved by an analogous argument.

(5): Symplectic forms:-

As usually formulated, Hamiltonian mechanics uses a non-degenerate antisymmetric 
bilinear form: i.e. $r=n$. So eq. 4.38 loses its bottom row and right column consisting of zero matrices, and reduces to the form of Section 4.3.1's naive symplectic matrix, eq. 4.10. Equivalently: eq. 4.37 reduces to eq. 4.18 .

Accordingly, we define: a symplectic form on a (real finite-dimensional) vector space $Z$ is a non-degenerate antisymmetric bilinear form $\omega$ on $Z: \omega: Z \times Z \rightarrow \mathbb{R}$. $Z$ is then called a symplectic vector space. It follows that $Z$ is of even dimension.

Besides, in Hamiltonian mechanics (as usually formulated) the vector space $Z$ is a product $V \times V^{*}$ of a vector space and its dual. Indeed, this was already suggested by:

(i) the fact in (3) of Section 2.2.2, that the canonical momenta $p_{i}:=\frac{\partial L}{\partial \dot{q}^{i}}$ transform as a 1 -form, and

(ii) Section 4.3.1's discussion of the one-form field $\nabla H$ determining a vector field $X_{H}$.

Thus we define the canonical symplectic form $\omega$ on $Z:=V \times V^{*}$ by

$$
\omega\left(\left(v_{1}, \alpha_{1}\right),\left(v_{2}, \alpha_{2}\right)\right):=\alpha_{2}\left(v_{1}\right)-\alpha_{1}\left(v_{2}\right) .
$$

So defined, $\omega$ is by construction a symplectic form, and so has the normal form given by eq. 4.10 .

Given a symplectic vector space $(Z, \omega)$, the natural question arises which linear maps $A: Z \rightarrow Z$ preserve the normal form given by eq. 4.10. It is straightforward to show that this is equivalent to $A$ preserving the form of Hamilton's equations (for any Hamiltonian); so that these maps $A$ are called canonical (or symplectic, or Poisson). But since (as I announced) this paper does not need details about the theory of canonical transformations, I will not go into details about this. Suffice it to say here the following.

$A: Z \rightarrow Z$ is symplectic iff, writing for the transpose (eq. 4.20) and using the second definition eq. 4.35 of $\sharp$, the following maps (both from $Z^{*}$ to $Z$ ) are equal:

$$
A \circ \omega^{\sharp} \circ \tilde{A}=\omega^{\sharp} ;
$$

or in matrix notation, with the matrix $\omega$ given by eq. 4.10 , and again writing $~{ }^{\text {for the }}$ transpose of a matrix

$$
A \omega \tilde{A}=\omega
$$

(Equivalent formulas are got by taking inverses. We get, respectively: $\tilde{A} \circ \omega^{b} \circ A=\omega^{b}$ and $\tilde{A} \omega A=\omega$.)

The set of all such linear symplectic maps $A: Z \rightarrow Z$ form a group, the symplectic group, written $\operatorname{Sp}(Z, \omega)$.

To sum up this Subsection:- We have, for a vector space $V, \operatorname{dim}(V)=n$, and $Z:=V \times V^{*}$ :

(i): the canonical symplectic form $\omega: Z \times Z \rightarrow \mathbb{R}$; with normal form given by eq. 4.10;

(ii): the associated linear map $\omega^{b}: Z \rightarrow Z^{*}$; which is an isomorphism, since $\omega$ is non-degenerate; 
(iii): the associated linear map $\omega^{\sharp}: Z^{*} \rightarrow Z$; which is an isomorphism, since $\omega$ is non-degenerate; and is the inverse of $\omega^{\text {b }}$; (cf. eq. 4.35).

We will see shortly that Hamiltonian mechanics takes $V$ to be the tangent space $T_{q}$ at a point $q \in Q$, so that $Z$ is $T_{q} \times T_{q}^{*}$, i.e. the tangent space to the space $\Gamma$ of the $q$ s and $p$ s.

\section{$5 \quad$ Poisson brackets and Noether's theorem}

We have seen how a single scalar function $H$ on phase space $\Gamma$ determines the evolution of the system via a combination of partial differentiation (the gradient of $H$ ) with the symplectic matrix. We now express these ideas in terms of Poisson brackets.

For our purposes, Poisson brackets will have three main advantages; which will be discussed in the following order in the Subsections below. Poisson brackets:

(i) give a neat expression for the rate of change of any dynamical variable;

(ii) give a version of Noether's theorem which is more simple and powerful (and even easier to prove!) than the Lagrangian version; and

(iii) lead to the generalized Hamiltonian framework mentioned in Section 6.8. All three advantages arise from the way the Poisson bracket encodes the way that a scalar function determines a (certain kind of) vector field.

\subsection{Poisson brackets introduced}

The rate of change of any dynamical variable $f$, taken as a scalar function on phase space $\Gamma, f(q, p) \in \mathbb{R}$, is given (with summation convention) by

$$
\frac{d f}{d t}=\dot{q}^{i} \frac{\partial f}{\partial q^{i}}+\dot{p}_{i} \frac{\partial f}{\partial p_{i}} .
$$

(If $f$ is time-dependent, $f:(q, p, t) \in \Gamma \times \mathbb{R} \mapsto f(q, p, t) \in \mathbb{R}$, the right-hand-side includes a term $\frac{\partial f}{\partial t}$. But on analogy with how our discussion of Lagrangian mechanics imposed scleronomic constraints, a time-independent work-function etc., we here set aside the time-dependent case.) Applying Hamilton's equations, this is

$$
\frac{d f}{d t}=\frac{\partial H}{\partial p_{i}} \frac{\partial f}{\partial q^{i}}-\frac{\partial H}{\partial q^{i}} \frac{\partial f}{\partial p_{i}} .
$$

This suggests that we define the Poisson bracket of any two such functions $f(q, p), g(q, p)$ by

$$
\{f, g\}:=\frac{\partial f}{\partial q^{i}} \frac{\partial g}{\partial p_{i}}-\frac{\partial f}{\partial p_{i}} \frac{\partial g}{\partial q^{i}}
$$

so that the rate of change of $f$ is given by

$$
\frac{d f}{d t}=\{f, H\} .
$$


In terms of the $2 n$ coordinates $\xi^{\alpha}$ (eq. 4.6) and the matrix elements $\omega^{\alpha \beta}$ of $\omega$ (eq. 4.13), we can write eq. 5.2 as

$$
\frac{d f}{d t}=\left(\partial_{\alpha} f\right) \dot{\xi}^{\alpha}=\left(\partial_{\alpha} f\right) \omega^{\alpha \beta}\left(\partial_{\beta} H\right) ;
$$

and so we can define the Poisson bracket by

$$
\{f, g\}:=\left(\partial_{\alpha} f\right) \omega^{\alpha \beta}\left(\partial_{\beta} g\right) \equiv \frac{\partial f}{\partial \xi^{\alpha}} \omega^{\alpha \beta} \frac{\partial g}{\partial \xi^{\beta}}
$$

In matrix notation: writing the naive gradients of $f$ and of $g$ as column vectors $\nabla f$ and $\nabla g$, and writing for transpose, we have at any point $z=(q, p) \in \Gamma$ :

$$
\{f, g\}(z)=\tilde{\nabla} f(z) \cdot \omega \cdot \nabla g(z)
$$

With these definitions of the Poisson bracket, we readily infer the following five results. (Later discussion will bring out the significance of some of these; in particular, Section 6.8 will take some of them to jointly define a primitive Poisson bracket for a generalized Hamiltonian mechanics.)

(1): Since the Poisson bracket is antisymmetric, $H$ itself is a constant of the motion:

$$
\frac{d H}{d t}=\{H, H\} \equiv 0 \text {. }
$$

(2): The Poisson bracket of a product is given by "Leibniz's rule": i.e. for any three functions $f, g, h$, we have

$$
\{f, h \cdot g\}=\{f, h\} \cdot g+h \cdot\{f, g\} \text {. }
$$

(3): Taking the Poisson bracket as itself a dynamical variable, its time-derivative is given by a "Leibniz rule"; i.e. the Poisson bracket behaves like a product:

$$
\frac{d}{d t}\{f, g\}=\left\{\frac{d f}{d t}, g\right\}+\left\{f, \frac{d g}{d t}\right\} .
$$

(4): The Jacobi identity (easily deduced from (3)):

$$
\{\{f, h\}, g\}+\{\{g, f\}, h\}+\{\{h, g\}, f\}=0 .
$$

(5): The Poisson brackets for the $q \mathrm{~s}, p \mathrm{~s}$ and $\xi_{\mathrm{s}}$ are:

$$
\begin{array}{r}
\left\{\xi^{\alpha}, \xi^{\beta}\right\}=\omega^{\alpha \beta} ; \text { i.e. } \\
\left\{q^{i}, p_{j}\right\}=\delta_{j}^{i}, \quad\left\{q^{i}, q^{j}\right\}=\left\{p_{i}, p_{j}\right\}=0 .
\end{array}
$$

Eq. 5.13 is very important, both for general theory and for problem-solving. The reason is that preservation of these Poisson brackets, by a smooth transformation of 
the $2 n$ variables $(q, p) \rightarrow(Q(q, p), P(q, p))$, is necessary and sufficient for the transformation being canonical. Besides, in this equivalence 'canonical' can be understood both in the usual elementary sense of preserving the form of Hamilton's equations, for any Hamiltonian function, and in the geometric sense of preserving the symplectic form (explained in (5) of Section 4.3.3, and for manifolds in Section 6).

Note here that, as the phrase 'for any Hamiltonian function' brings out, the notion of a canonical transformation is independent of the forces on the system as encoded in the Hamiltonian. That is: the notion is a matter of $\Gamma$ 's geometry - as we will emphasise in Section 6.

But (as I announced in Section 4.1) I will not need to go into many details about canonical transformations, essentially because this paper does not aim to survey the whole of Hamiltonian mechanics, or even all that can be said about reducing problems, e.g. by finding simplifying canonical transformations. It aims only to survey the way that symmetries and conserved quantities effect such reductions. In the rest of this Subsection, I begin describing Poisson brackets' role in this, in particular Noether's theorem. But the description can only be completed once we have the geometric perspective on Hamiltonian mechanics, i.e. in Section 6.5.

\subsection{Hamiltonian vector fields}

Section 4.3.1 described how the symplectic matrix enabled the scalar function $H$ on $\Gamma$ to determine a vector field $X_{H}$. The previous Subsection showed how the Poisson bracket expressed any dynamical variable's rate of change along $X_{H}$. We now bring these ideas together, and generalize.

Recall that a vector $X$ at a point $x$ of a manifold $M$ can be identified with a directional derivative operator at $x$ assigning to each smooth function $f$ defined on a neighbourhood of $x$ its directional derivative along any curve that has $X$ as its tangent vector. Thus recall the Lagrangian definition of the dynamical vector field, eq. 2.8 in Section 2.2. Similarly here: the dynamical vector field $X_{H}=: D$ is a derivative operator on scalar functions, which can be written in terms the Poisson bracket:

$$
D:=X_{H}=\frac{d}{d t}=\dot{q}^{i} \frac{\partial}{\partial q^{i}}+\dot{p}_{i} \frac{\partial}{\partial p_{i}}=\frac{\partial H}{\partial p_{i}} \frac{\partial}{\partial q^{i}}-\frac{\partial H}{\partial q^{i}} \frac{\partial}{\partial p_{i}}=\{\cdot, H\} .
$$

But this point applies to any smooth scalar, $f$ say, on $\Gamma$. That is: although we think of $H$ as the energy that determines the real physical evolution, the mathematics is of course the same for such an $f$. So any such function determines a vector field, $X_{f}$ say, on $\Gamma$ that generates what the evolution "would be if $f$ was the Hamiltonian". Thinking of the integral curves as parametrized by $s$, we have

$$
X_{f}=\frac{d}{d s}=\{\cdot, f\} .
$$

$X_{f}$ is called the Hamiltonian vector field of (for) $f$; just as, for the physical Hamiltonian, $f \equiv H$, Section 4.3.1 called $X_{H}$ 'the Hamiltonian vector field'. 
The notion of a Hamiltonian vector field will be crucial for what follows, not least for Noether's theorem in the very next Subsection. For the moment, we just make two remarks which we will need later.

So every scalar $f$ determines a Hamiltonian vector field $X_{f}$. But note that the converse is false: not every vector field $X$ on $\Gamma$ is the Hamiltonian vector field of some scalar. For a vector field (equations of motion) $X$, with components $X^{\alpha}$ in the coordinates $\xi^{\alpha}$ defined by eq. 4.6

$$
\dot{\xi}^{\alpha}=X^{\alpha}(\xi)
$$

there need be no scalar $H: \Gamma \rightarrow \mathbb{R}$ such that, as required by eq. 4.13 ,

$$
X^{\alpha}=\omega^{\alpha \beta} \partial_{\beta} H \text {. }
$$

This is the same point as in (ii) of Section 4.2.3: that Hamilton's equations have the special feature that all the right hand sides are, up to a sign, partial derivatives of a single function $H$ - a feature that underpins the possibility of expressing the equations of motion by variational principles.

We also need to note under what condition is a vector field $X$ Hamiltonian; (this will bear on Noether's theorem). The answer is: $X$ is locally Hamiltonian, i.e. there is locally a scalar $f$ such that $X=X_{f}$, iff $X$ generates a one-parameter family of canonical transformations. We will give a modern geometric proof of this in Section 6.5. For the moment, we only need to note, as at the end of Section 5.1, that here 'canonical transformation' can be understood in the usual elementary sense as a transformation of $\Gamma$ that preserves the form of Hamilton's equations (for any Hamiltonian); or equivalently, as preserving the Poisson bracket; or equivalently, as preserving the symplectic form (to be defined for manifolds, in Section 6).

\subsection{Noether's theorem}

\subsubsection{An apparent "one-liner", and three claims}

In the Hamiltonian framework, the core of the proof of Noether's theorem is very simple; as follows. The Poisson bracket is obviously antisymmetric. So for any scalar functions $f$ and $H$, we have

$$
X_{f}(H) \equiv \frac{d H}{d s} \equiv\{H, f\}=0 \quad \text { iff } \quad 0=\{f, H\}=X_{H}(f) \equiv D(f) .
$$

In words: $H$ is constant under the flow of the vector field $X_{f}$ (i.e. under what the evolution would be if $f$ was the Hamiltonian) iff $f$ is constant under the dynamical flow $X_{H} \equiv D$.

This "one-liner" is the Hamiltonian version of Noether's theorem! There are three claims here. The first two relate back to the Lagrangian version of the theorem. The 
third is about the definition of a (continuous) symmetry for a Hamiltonian system, and so about how we should formulate the Hamiltonian version of Noether's theorem. I will state all three claims, but in this Subsection justify only the first two. For it will be convenient to postpone the third till after we have introduced some modern geometry (Section 6.5).

First, for eq. 5.18 to deserve the name 'Noether's theorem', I need to show that it encompasses Section 3's Lagrangian version of Noether's theorem (despite the trivial proof!).

Second, in order to justify my claim that the Hamiltonian version of Noether's theorem is more powerful than the Lagrangian version, I need to show that eq. 5.18 says more than that version, i.e. that it covers more symmetries.

To state the third claim, note first that we expect a Hamiltonian version of Noether's theorem to say something like: to every continuous symmetry of a Hamiltonian system, there corresponds a conserved quantity. Here, we expect a 'continuous symmetry' to be defined by a vector field on $\Gamma$ (or by its flow). Indeed, a symmetry of a Hamiltonian system is usually defined as a transformation of $\Gamma$ that:

(1) is canonical; (a condition independent of the forces on the system as encoded in the Hamiltonian: a matter of $\Gamma$ 's intrinsic geometry); and also

(2) preserves the Hamiltonian function; (a condition obviously dependent on the Hamiltonian).

Accordingly, a continuous symmetry is defined as a vector field on $\Gamma$ that generates a one-parameter family of such transformations; (or as such a field's flow, i.e. as the family itself).

But with this definition of 'continuous symmetry' (of a Hamiltonian system), eq. 5.18 seems to suffer from two lacunae, if taken to express Noether's theorem, that to every continuous symmetry there corresponds a conserved quantity. Agreed, the rightward implication of eq. 5.18 provides, for a vector field $X_{f}$ with property (2), the conserved quantity $f$. But there seem to be two lacunae:

(a): eq. 5.18 is silent about whether $X_{f}$ has property (1), i.e. generates canonical transformations.

(b): eq. 5.18 considers only Hamiltonian vector fields, i.e. vector fields $X$ induced by some $f, X=X_{f}$. But as noted at the end of Section 5.2, there are countless vector fields on $\Gamma$ that are not Hamiltonian. If such a field could be a continuous symmetry, eq. 5.18's rightward implication would fall short of saying that to every continuous symmetry, there corresponds a conserved quantity.

So the third claim I need is that these lacunae are illusory. In fact, a single result will deal with both (a) and (b). Namely, it will suffice to show that a vector field $X$ on $\Gamma$ has property (1), i.e. generates canonical transformations, iff it is Hamiltonian, i.e. induced by some $f, X=X_{f}$. But I postpone showing this till we have more modern geometry in hand; cf. Section 6.5. 


\subsubsection{The relation to the Lagrangian version}

On the other hand, we can establish the first two claims with the elementary apparatus so far developed. I will concentrate on justifying the first claim; that will also make the second claim clear.

For the first claim, we need to show that:

(i): to any variational symmetry of the Lagrangian $L$, i.e. a vector field $X$ on $Q$ obeying eq. 3.6, there corresponds a vector field $X_{f}$ on $\Gamma$ for which $X_{f}(H)=0$; and

(ii): the correspondence in (i) is such that the scalar $f$ can be taken to be (the Hamiltonian version of) the momentum $p_{X}$ conjugate to $X$, defined by eq. 3.12 (or geometrically, by 3.31).

It will be clearest to proceed in two stages.

(A): First, I will show (i) and (ii).

(B): Then I will discuss how (A) relates to the usual definition of a symmetry of a Hamiltonian system.

(A): The easiest way to show (i) and (ii) is to use the fact discussed after eq. 3.20, that every variational symmetry $X$ arises, around a point where it is non-zero, from a cyclic coordinate in some local system of coordinates. (Recall that this follows from the basic "rectification" theorem securing the local existence and uniqueness of solutions of ordinary differential equations.) That is, there is some coordinate system $(q)$ on some open subset of $X$ 's domain of definition on $Q$ such that

(a): $X$ being a variational symmetry is equivalent to $q^{n}$ being cyclic, i.e. $\frac{\partial L}{\partial q^{n}}=0$;

(b): the momentum $p_{X}$, which the Lagrangian theorem says is conserved, is the elementary generalized momentum $p_{n}:=\frac{\partial L}{\partial \dot{q}^{n}}$.

So suppose given a variational symmetry $X$, and a coordinate system $(q)$ satisfying (a)-(b). Now we recall that the Legendre transformation, i.e. the transition between Lagrangian and Hamiltonian frameworks, does not "involve the dependence on the $q \mathrm{~s}$ ". More precisely, we recall eq. 4.8, $\frac{\partial H}{\partial q^{n}}=-\frac{\partial L}{\partial q^{n}}$. Now consider $p_{n}: \Gamma \rightarrow \mathbb{R}$. This $p_{n}$ will do as the function $f$ required in (i) and (ii) above, since

$$
X_{p_{n}}(H) \equiv\left\{H, p_{n}\right\}=\frac{\partial H}{\partial q^{n}}=-\frac{\partial L}{\partial q^{n}}=0 .
$$

Applying eq. 5.18 to eq. 5.19 , we deduce that $p_{n}$, i.e. the $p_{X}$ of the Lagrangian theorem, is conserved.

(Hence my remark after eq. 4.8, that the elementary result that $p_{n}$ is conserved iff $q^{n}$ is cyclic, underpins the Hamiltonian version of Noether's theorem; just as the corresponding Lagrangian result underpins the Lagrangian version of Noether's theorem: cf. discussion after eq. 3.20.)

(B): I agree that this simple proof seems suspiciously simple. Besides, the suspicion grows when you notice that my argument in (A) has not used a definition of a symmetry, in particular a continuous symmetry, of a Hamiltonian system (contrast Section 3.2). As discussed in Section 5.3.1, we expect a Hamiltonian version of Noether's theorem 
to say 'to every continuous symmetry of a Hamiltonian system there corresponds a conserved quantity'; where a continuous symmetry is a vector field that (1) generates canonical transformations and (2) preserves the Hamiltonian. So the argument in (A) is suspicious since, although eq. 5.19, or the left hand side of eq. 5.18, obviously expresses property (2), i.e. preserving the Hamiltonian, the argument in (A) seems to nowhere use property (1), i.e. the symmetry generating canonical transformations.

But in fact, all is well. The reason why lies in the fact mentioned in (i), (a) of Section 4.1: that every point transformation (together with its lift to $T Q$ ) defines a corresponding canonical transformation on $T^{*} Q$. That is to say: property (1) is secured by the fact that the Lagrangian Noether's theorem of Section 3 is restricted to symmetries induced by point transformations.

In other words, in terms of the vector field (variational symmetry) $X$ given us by (a) in (A) above: one can check that $X$ defines a vector field on $\Gamma$ (equivalently: a oneparameter family of transformations on $\Gamma$ ) that is canonical, i.e. preserves Hamilton's equations or equivalently the symplectic form. Indeed, one can easily check that, once we rectify the Lagrangian variational symmetry $X$, so that it generates the rectified one-parameter family of point transformations: $q_{i}=$ const, $i \neq n ; q_{n} \mapsto q_{n}+\epsilon$, the vector field that $X$ defines on $\Gamma$ is precisely the field $X_{p_{n}}$ chosen above. ${ }^{17}$

Finally, the discussion in (B) also vindicates the second claim in Section 5.3.1: that the Hamiltonian version of Noether's theorem, eq. 5.18, says more than the Lagrangian version, i.e. covers more symmetries. This follows from the fact (announced in (i) (b) of Section 4.1) that there are canonical transformations not induced by a point transformation (together with its lift).

In elementary discussions, this is often expressed in terms of canonical transformations being allowed to "mix" the $q \mathrm{~s}$ and $p s$. But a more precise, and geometric, statement is the result announced at the end of Section 5.2 (whose proof is postponed to Section 6.5): that the condition for a vector field on $\Gamma$ to generate a one-parameter family of canonical transformations is merely that it be a Hamiltonian vector field. That is: for any scalar $f: \Gamma \rightarrow \mathbb{R}$, the vector field $X_{f}$ generates such a family.

In this sense, canonical transformations are two a penny (also known as: a dime a dozen!). So it is little wonder that most discussions emphasise the other condition, i.e. property (2): that $X_{f}$ preserve the Hamiltonian, $X_{f}(H)=0$. Only very special $f_{\mathrm{s}}$ will satisfy $X_{f}(H)=0$; and if we are given $H$ (in certain coordinates $q, p$ ), it can be very hard to find (the coordinate expression of) such an $f$.

Indeed, when Jacobi first propounded the theory of canonical transformations, in his Lectures on Dynamics (1842), he was of course aware of this. Accordingly, he pointed out that in theoretical mechanics, it was often more fruitful to first consider an $f$ (equivalently: a canonical transformation), and then cast about for a Hamiltonian that it preserved. He wrote: 'The main difficulty in integrating a given differential

\footnotetext{
${ }^{17}$ Details about point transformations on $Q$ defining a canonical transformation on $T^{*} Q$, and lifting the vector field $X$ to $\Gamma$, can be found: (i) using traditional terms, in Goldstein et al. (2002: 375-376) and Lanczos (1986: Chapter VII.2); (ii) using modern geometric terms (as developed in Section 6), in Abraham and Marsden (1978: Sections 3.2.10-3.2.12) and Marsden and Ratiu (1999: Sections 6.3-6.4).
} 
equation lies in introducing convenient variables, which there is no rule for finding. Therefore we must travel the reverse path and after finding some notable substitution, look for problems to which it can be successfully applied'; (quoted in Arnold (1989, p. 266)). The fact that Jacobi solved many previously intractable problems bears witness to the power of this strategy, and of his theory of canonical transformations.

We can sum up this Subsection in two comments:-

(1) In Hamiltonian mechanics, Noether's theorem is a biconditional, an 'iff' statement. Not only does a Hamiltonian symmetry - i.e. a vector field $X$ on $\Gamma$ that generates canonical transformations (equivalently: preserves the symplectic form, or the Poisson bracket) and preserves the Hamiltonian, $X(H)=0$-provide a constant of the motion. Also, given a constant of the motion $f: \Gamma \rightarrow \mathbb{R}$, there is a symmetry of the Hamiltonian, viz. the vector field $X_{f}$. (Or if one prefers the integral notion of symmetry: the flow of $X_{f}$ ). This converse implication, from constant to symmetry, contrasts with the Lagrangian framework; cf. the end of Section 3.4.1.

(2) In elementary Hamiltonian mechanics, Noether's theorem has a very simple one-line proof, viz. eq. 5.18.

Later, we will return to Noether's theorem. Section 6.5 will justify the third claim of Section 5.3.1, by showing that a vector field generates a one-parameter family of canonical transformations iff it is a Hamiltonian vector field. Meanwhile, we end Section 5 with a comment about "iterating" Noether's theorem, and the distinction between such an iteration and the idea of complete integrability.

\subsection{Glimpsing the "complete solution"}

Suppose we "iterate" Noether's theorem. That is: suppose there are several (continuous) symmetries of the Hamiltonian and so several constants of the motion. Each will confine the system's time-evolution to a $(2 n-1)$-dimensional hypersurface of $\Gamma$. In general, the intersection of $k$ such surfaces will be a hypersurface of dimension $2 n-k$ (i.e. of co-dimension $k$ ); to which the motion is therefore confined. The theory of symplectic reduction (Butterfield 2006) describes how to do a "quotiented dynamics" in this general situation. Here, I just remark on one aspect; which will not be developed in the sequel.

Locally, the rectification theorem secures, for any system, not just several constants of the motion, but "all you could ask for". Applying the theorem (eq. 3.21 and 3.22) to the Hamiltonian vector field $X_{H}$ on $\Gamma$, we infer that locally there are coordinates $\xi^{\alpha}$ (maybe very hard to find!) in which $X_{H}$ has $2 n-1$ components that vanish throughout the neighbourhood, while the other component is 1 :

$$
X_{H}^{\alpha}=0 \text { for } \alpha=1,2, \ldots, 2 n-1 ; X_{H}^{2 n}=1 .
$$

So the coordinates $\xi^{\alpha}, \alpha=1, \ldots, 2 n-1$, form $2 n-1$ constants of the motion. They are functionally independent, and all other constants of the motion are functions of them; (cf. point (ii) after eq. 3.22). So the motion is confined to the one-dimensional 
intersection of the $2 n-1$ hypersurfaces, each of co-dimension 1 . That is to say, it is confined to the curve given by: $\xi^{\alpha}=$ const, $\alpha=1, \ldots, 2 n-1, \xi^{2 n}=t$.

To this, Noether's theorem eq. 5.18 adds the physical idea that each such constant of the motion defines a vector field $X_{\xi^{\alpha}}$ that generates a symmetry of the Hamiltonian:

$$
X_{\xi^{\alpha}}(H)=0, \text { for } \alpha=1,2, \ldots, 2 n-1 .
$$

In this local sense, the "complete solution" of any Hamiltonian system lies in the local constants of the motion, or equivalently the local symmetries of its Hamiltonian $H$.

To sum up: locally, any Hamiltonian system is "completely integrable". But the scare-quotes here are a reminder that these phrases are usually used with other, stronger, meanings: either that there are $2 n-1$ global constants of the motion or that the system is completely integrable in the sense of Liouville's theorem.

\section{A geometrical perspective}

In this final Section, we develop the modern geometric description of Hamiltonian mechanics. We will build especially on Sections 4.3; one main aim will of course be to complete the discussion of Noether's theorem, begun in Section 5.3.

There will be eight Subsections. First, we introduce the cotangent bundle $T^{*} Q$. Then we collect what we will need about forms. Then we can show that any cotangent bundle is a symplectic manifold. This enables us to formulate Hamilton's equations geometrically; and to complete the discussion of Noether's theorem. Then we report Darboux's theorem, and its relation to reduction of problems. Then we return to the Lagrangian framework, by sketching the geometric formulation of the Legendre transformation. Finally, we "glimpse the landscape ahead" by mentioning the more general framework for Hamiltonian mechanics that uses Poisson manifolds.

\subsection{Canonical momenta are one-forms: $\Gamma$ as $T^{*} Q$}

So far we have treated the phase space $\Gamma$ informally: saying just that it is a $2 n$ dimensional space coordinatized by the $q s$, a smooth coordinate system on the configuration manifold $Q$, and the $p$ s, which are canonical momenta $\frac{\partial L}{\partial \dot{q}^{i}}$. But we also saw in (3) of Section 2.2.2 that at each point $q \in Q$, the $p_{i}$ transform as a 1-form (eq. 2.12). Accordingly we now take the physical state of the system to be a point in the cotangent bundle $T^{*} Q$, the $2 n$-dimensional manifold whose points are pairs $(q, p)$ with $q \in Q, p \in T_{q}^{*}$.

I stress that from now on, the symbol $p$ has a (fruitful!) ambiguity, between "dynamics" and "kinematics/geometry". For $p$ represents both:

(A) the conjugate momentum $\frac{\partial L}{\partial \dot{q}}$, which of course depends on the choice of $L$; and

(B) a point in a fibre $T_{q}^{*}$ of the cotangent bundle $T^{*} Q$ (i.e. a 1-form or covector); 
or relatedly: the components $p_{i}$ of such a 1-form: notions that are independent of any choice of a Lagrangian or Hamiltonian.

In more detail:-

(A): Recall that in the Lagrangian framework, the basic equations (eq. 2.1, or Newton's second law!) being second-order in time prompts us to take the initial $q$ and $\dot{q}$ as chosen independently, with $L$ (encoding the forces on the system) then determining the evolution (the Lagrangian dynamical vector field $D$ ) - and so also determining the actual "realized" value of $\dot{q}$ at other times as a function of $q$, and so ultimately, of $t$. Similarly here: Newton's second law being second-order in time prompts us to take the initial $q$ and $p$ as independent, with $H$ (encoding the forces on the system) then determining the evolution (the Hamiltonian dynamical vector field $D$ ) -and so also determining the actual value of $p$ at other times as a function of $q$, and so ultimately, of $t$. Besides, by passing via the Legendre transformation back to the Lagrangian framework, one can check that the later actual value of $p$ is determined to equal $\frac{\partial L}{\partial \dot{q}}$.

(B): But $p$ also represents any 1-form (so that $p_{i}$ represents the 1-form's coordinates). Here, we need to recall three points:-

(i): A local coordinate system (a chart) on $Q$ defines a basis in the tangent space $T_{q}$ at any point $q$ in the chart's domain. As usual, I write the chart's coordinate functions as $q^{i}$. So I shall temporarily denote the chart by $[q]$, so that there are coordinate functions $q^{i}: \operatorname{dom}([q]) \rightarrow \mathbb{R}$. I write elements of the coordinate basis as usual, as $\frac{\partial}{\partial q^{i}}$.

(ii): The chart $[q]$ thereby also defines a dual basis $d q^{i}$ in the cotangent space $T_{q}^{*}$ at any $q \in \operatorname{dom}([q])$.

(Here I recall, en passant, that the isomorphism at each $q$ between $T_{q}$ and $T_{q}^{*}$, that maps the basis element $\frac{\partial}{\partial q^{i}} \in T_{q}$ to the one-form $d q^{i}$ in the dual basis, is basisdependent. A different basis $\frac{\partial}{\partial q^{\prime i}}$ would give a different isomorphism. Cf. the discussion in (1) of Section 4.3.3.)

(iii): Putting (i) and (ii) together: the chart $[q]$ thereby also induces a local coordinate system on a neighbourhood of the cotangent bundle around any point $(q, p) \in T^{*} Q$ with $q \in \operatorname{dom}([q])$ and $p \in T_{q}^{*}$.

Putting (i)-(iii) together: the coordinates of any point $(q, p)$ in $T^{*} Q$ in such a coordinate system are usually also written as $(q, p)$. That is: $p$ is used for the components of any 1 -form, in the basis $d q^{i}$ dual to a coordinate basis $\frac{\partial}{\partial q^{i}}$. So, similarly to (i) above: I will write this induced chart on $T^{*} Q$ as $[q, p]$.

(C): Taken together, points (A) and (B) prompt a question:

Why should an evolution from an arbitrary initial state $\in T^{*} Q$ have the property that:-

if we choose to express

(i) its configuration, $q_{0}$ say, in terms of an arbitrary initial coordinate system

$[q]$ on $Q$, and

(ii) its momenta $\frac{\partial L}{\partial \dot{q}}$ in terms of the basis $d q$ dual to the coordinate basis $\frac{\partial}{\partial q}$ at $q_{0}:-$ 
then

the states at a later time $t$ have their momenta - which the Lagrangian framework tells us must be $\frac{\partial L}{\partial \dot{q}}(\mathrm{cf}$. (A)) - equal to their components in the dual basis to the later coordinate basis, i.e. the coordinate basis $\frac{\partial}{\partial q}$ at the later configuration $q_{t}$ ?

In short: why should the state's components in the dual basis of any coordinate basis continue to be equal, as dynamical evolution goes on, to the values of canonical momenta i.e. $\frac{\partial L}{\partial \dot{q}}$ ?

A good question. The short answer lies in combining Hamilton's equations for the time-derivative of the $p_{i}$ (eq. 4.5) with Lagrange's equations, and with the fact that the partial derivatives with respect to $q^{i}$ of the Hamiltonian and Lagrangian, $H$ and $L$, are negatives of each other (eq. 4.8). Thus we have:

$$
\dot{p}_{i}=-\frac{\partial H}{\partial q^{i}}=\frac{\partial L}{\partial q^{i}}=\frac{d}{d t}\left(\frac{\partial L}{\partial \dot{q}^{i}}\right)
$$

From this it is clear that for any coordinate system, if at $t_{0}, p_{i}$ is chosen to equal $\frac{\partial L}{\partial \dot{q}^{i}}$, then this will be so at later times. For eq. 6.1 forces their time-derivatives to be equal - and so also, their later values must be equal.

So much for the short answer. We will also get more insight into the relations between the Lagrangian and Hamiltonian frameworks in

(i) the fact, expounded in Section 6.3 below, that any cotangent bundle has a natural symplectic structure, independent of the specification of any Lagrangian or Hamiltonian function; and

(ii) some further details about the Legendre transformation, which is further discussed in Section 6.7.

\subsection{Forms, wedge-products and exterior derivatives}

As I said at the end of Section 4.3.2, this paper can largely avoid the theory of forms. For what follows (especially Section 6.5), I need to recall only:

(i) the idea of forms of various degrees, together comprising the exterior algebra, and equipped with operations of wedge-product and contraction (Section 6.2.1);

(ii) the ideas of differential forms, the exterior derivative, and of exact and closed forms (Section 6.2.2).

\subsubsection{The exterior algebra; wedge-products and contractions}

We begin by recalling some ideas of Sections 4.3.2 and 4.3.3. Let us again begin with the simplest possible case, $\mathbb{R}^{2}$, considered as a vector space: not as a manifold with a copy of itself as tangent space at each point. 
If $\alpha, \beta$ are covectors, i.e. elements of $\left(\mathbb{R}^{2}\right)^{*}$, we define their wedge-product, an antisymmetric bilinear form on $\mathbb{R}^{2}$, by

$$
\alpha \wedge \beta:(v, w) \in \mathbb{R}^{2} \times \mathbb{R}^{2} \mapsto(\alpha(v))(\beta(w))-(\alpha(w))(\beta(v)) \in \mathbb{R} .
$$

Let us write the standard basis elements of $\mathbb{R}^{2}$ as $\frac{\partial}{\partial q}$ and $\frac{\partial}{\partial p}$, with elements of $\mathbb{R}^{2}$ having components $(q, p)$ in this basis; and let us write the elements of the dual basis as $d q, d p$. Recalling the definition of the area form $A$, eq. 4.16, we deduce that $A$ is $d q \wedge d p$.

Similarly for $\mathbb{R}^{2 n}$. Recall that the symplectic matrix defines an antisymmetric bilinear form on $\mathbb{R}^{2 n}$ by eq. 4.18. The value on a pair $(q, p) \equiv\left(q^{1}, \ldots q^{n} ; p_{1}, \ldots, p_{n}\right),\left(q^{\prime}, p^{\prime}\right) \equiv$ $\left(q^{1}, \ldots q^{\prime n} ; p_{1}^{\prime}, \ldots, p_{n}^{\prime}\right)$ is the sum of the signed areas of the $n$ parallelograms formed by the projections of the vectors $(q, p),\left(q^{\prime}, p^{\prime}\right)$ onto the $n$ pairs of coordinate planes. This is a sum of $n$ wedge-products. That is to say: if we write the standard basis elements as $\frac{\partial}{\partial q^{i}}$ and $\frac{\partial}{\partial p_{i}}$, this form is $\omega:=\Sigma_{i} d q^{i} \wedge d p_{i}$. It has the action on $\mathbb{R}^{n} \times \mathbb{R}^{n}$ :

$$
\left(q^{i} \frac{\partial}{\partial q^{i}}+p_{i} \frac{\partial}{\partial p_{i}}, q^{\prime i} \frac{\partial}{\partial q^{i}}+p_{i}^{\prime} \frac{\partial}{\partial p_{i}}\right) \mapsto \sum_{i=1}^{n} q^{i} p_{i}^{\prime}-q^{\prime i} p_{i} .
$$

In general, if $V, W$ are two (real finite-dimensional) vector spaces, we define: $L(V, W)$ to be the vector space of linear maps from $V$ to $W ; L^{k}(V, W)$ to be the vector space of $k$-multilinear maps from $V \times V \times \ldots . \times V$ ( $k$ copies $)$ to $W$; and $L_{a}^{k}(V, W)$ to be the subspace of $L^{k}(V, W)$ consisting of (wholly) antisymmetric maps.

We then define $\Omega^{k}(V):=L_{a}^{k}(V, \mathbb{R})$ for $k=1,2, \ldots, \operatorname{dim}(V)$, so that $\Omega^{1}(V)=V^{*}$. We also set $\Omega^{0}(V):=\mathbb{R}$. $\Omega^{k}(V)$ is called the space of (exterior) $k$-forms on $V$. If $\operatorname{dim}(V)=n$, then $\operatorname{dim}\left(\Omega^{k}(V)\right)=\left(\begin{array}{c}n \\ k\end{array}\right)$.

The wedge-product, as defined above, can be extended to be an operation that defines, for $\alpha \in \Omega^{k}(V), \beta \in \Omega^{l}(V)$, an element $\alpha \wedge \beta \in \Omega^{k+l}(V)$. We can skip the details: suffice it to say that the idea is to take tensor products as in (3) of Section 4.3.3, and anti-symmetrize.

But to complete our discussion of Noether's theorem (in Section 6.5), we will need the definition of the contraction, (also known as: interior product), of a $k$-form $\alpha \in$ $\Omega^{k}(V)$ with a vector $v \in V$. We shall write this as $\mathbf{i}_{v} \alpha$. (It is also written with a hook notation.) We define the contraction $\mathbf{i}_{v} \alpha$ to be the $(k-1)$-form given by:

$$
\mathbf{i}_{v} \alpha\left(v_{2}, \ldots, v_{k}\right):=\alpha\left(v, v_{2}, \ldots, v_{k}\right) .
$$

It follows, for example, that contraction distributes over the wedge-product modulo a sign, in the following sense. If $\alpha$ is a $k$-form, and $\beta$ a 1 -form, then

$$
\mathbf{i}_{v}(\alpha \wedge \beta)=\left(\mathbf{i}_{v} \alpha\right) \wedge \beta+(-1)^{k} \alpha \wedge\left(\mathbf{i}_{v} \beta\right) .
$$

The direct sum of the vector spaces $\Omega^{k}(V), k=0,1,2, \ldots, \operatorname{dim}(V)=: n$, has dimension $2^{n}$. When this direct sum is considered as equipped with the wedge-product $\wedge$ and contraction $\mathbf{i}$, it is called the exterior algebra of $V$, written $\Omega(V)$. 


\subsubsection{Differential forms; the exterior derivative; the Poincaré Lemma}

We extend the discussion given in Section 6.2.1 to a manifold $M$ of dimension $n$, taking all the tangent spaces $T_{x}$ at $x \in M$ as copies of the vector space $V$, and requiring fields of forms to be suitably smooth.

We begin by saying that a (smooth) scalar function $f: M \rightarrow \mathbb{R}$ is a 0 -form field. Its differential or gradient, $d f$, as defined by its action on all vector fields $X$, viz. mapping them to $f$ 's directional derivative along $X$

$$
d f(X):=X(f)
$$

is a 1-form (covector) field, called a differential 1-form.

The set $\mathcal{F}(M)$ of all smooth scalar functions forms an (infinite-dimensional) vector space, indeed a ring, under pointwise operations. We write the set of vector fields on $M$ as $\mathcal{X}(M)$, or as $\mathcal{T}_{0}^{1}(M)$; and the set of covector fields, i.e. differential 1-forms, on $M$ as $\mathcal{X}^{*}(M)$, or as $\mathcal{T}_{1}^{0}(M)$. (So superscripts indicate the contravariant order, and subscripts the covariant order.)

Accordingly, we define: $\Omega^{0}(M):=\mathcal{F}(M) ; \Omega^{1}(M)=\mathcal{T}_{1}^{0}(M)$; and so on. In short: $\Omega^{k}(M)$ is the set of smooth fields of exterior $k$-forms on the tangent spaces of $M$.

The wedge-product, as defined in Section 6.2.1, can be extended to the various $\Omega^{k}(M)$. We form the direct sum of the (infinite-dimensional) vector spaces $\Omega^{k}(M), k=$ $0,1,2, \ldots, \operatorname{dim}(V)=: n$, and consider it as equipped with this extended wedge-product. We call it the algebra of exterior differential forms on $M$, written $\Omega(M)$.

Similarly, contraction, as defined in Section 6.2.1, can be extended to $\Omega(M)$. On analogy with eq. 6.4, we define, for $\alpha$ a $k$-form field on $M$, and $X$ a vector field on $M$, the contraction $\mathbf{i}_{X} \alpha$ to be the $(k-1)$-form given, at each point $x \in M$, by:

$$
\mathbf{i}_{X} \alpha(x):\left(v_{2}, \ldots, v_{k}\right) \mapsto \alpha(x)\left(X(x), v_{2}, \ldots, v_{k}\right) \in \mathbb{R} .
$$

The exterior derivative is a differential operator on $\Omega(M)$ that maps a $k$-form field to a $(k+1)$-form field. In particular, it maps a scalar $f$ to its differential (gradient) $d f$. Indeed, it is the unique map from the $k$-form fields to the $(k+1)$-form fields $(k=1,2, \ldots, n)$ that generalizes the elementary notion of gradient $f \mapsto d f$, subject to certain natural conditions.

To be precise: one can show that there is a unique family of maps $d^{k}: \Omega^{k}(M) \rightarrow$ $\Omega^{k+1}(M)$, all of which, for simplicity, we write as $\mathbf{d}$, such that:

(a): If $f \in \mathcal{F}(M), \mathbf{d}(f)=d f$.

(b): $\mathbf{d}$ is $\mathbb{R}$-linear; and distributes across the wedge-product, modulo a sign. That is: for $\alpha \in \Omega^{k}(M), \beta \in \Omega^{l}(M), \mathbf{d}(\alpha \wedge \beta)=(\mathbf{d} \alpha) \wedge \beta+(-1)^{k} \alpha \wedge(\mathbf{d} \beta)$. (Cf. eq. 6.5.)

(c): $\mathbf{d}^{2}:=\mathbf{d} \circ \mathbf{d} \equiv 0$; i.e. for all $\alpha \in \Omega^{k}(M) d^{k+1} \circ d^{k}(\alpha) \equiv 0$. (This condition looks strong, but is in fact natural. For its motivation, it must here suffice to say that it generalizes the fact in elementary vector calculus, that the curl of any gradient is zero: $\nabla \wedge(\nabla f) \equiv 0$. 
(d): $\mathbf{d}$ is a local operator; i.e. for any $x \in M$ and any $k$-form $\alpha, \mathbf{d} \alpha(x)$ depends only on $\alpha$ 's restriction to any open neighbourhood of $x$; more precisely, we define for any open set $U$ of $M$, the vector space $\Omega^{k}(U)$ of $k$-form fields on $U$, and then require that

$$
\mathbf{d}\left(\left.\alpha\right|_{U}\right)=\left.(\mathbf{d} \alpha)\right|_{U}
$$

To express $\mathbf{d}$ in terms of coordinates: if $\alpha \in \Omega^{k}(M)$, i.e. $\alpha$ is a $k$-form on $M$, given in coordinates by

$$
\alpha=\alpha_{i_{1} \ldots i_{k}} d x^{i_{1}} \wedge \cdots \wedge d x^{i_{k}} \quad\left(\text { sum on } i_{1}<i_{2}<\ldots<i_{k}\right),
$$

then one proves that the exterior derivative is

$$
\mathbf{d} \alpha=\frac{\partial \alpha_{i_{1} \ldots i_{k}}}{\partial x^{j}} d x^{j} \wedge d x^{i_{1}} \wedge \cdots \wedge d x^{i_{k}} \quad\left(\text { sum on all } j \text { and } i_{1}, \ldots<i_{k}\right),
$$

We define $\alpha \in \Omega^{k}(M)$ to be:

exact if there is a $\beta \in \Omega^{k-1}(M)$ such that $\alpha=\mathbf{d} \beta$; (cf. the elementary definition of an exact differential);

closed if $\mathbf{d} \alpha=0$.

It is immediate from condition (c) above, $\mathbf{d}^{2}=0$, that every exact form is closed. The converse is "locally true". This important result is the Poincaré Lemma; (and we will use it in Section 6.5's closing discussion of Noether's theorem).

To be precise: for any open set $U$ of $M$, we define (as in condition (d) above) the vector space $\Omega^{k}(U)$ of $k$-form fields on $U$. Then the Poincaré Lemma states that if $\alpha \in \Omega^{k}(M)$ is closed, then at every $x \in M$ there is a neighbourhood $U$ such that $\left.\alpha\right|_{U} \in \Omega^{k}(U)$ is exact.

We will also need (again, for Section 6.5's discussion of Noether's theorem) a useful formula relating the Lie derivative, contraction and the exterior derivative. Namely: Cartan's magic formula, which says that if $X$ is a vector field and $\alpha$ a $k$-form on a manifold $M$, then the Lie derivative of $\alpha$ with respect to $X$ (i.e. along the flow of $X$ ) is

$$
\mathcal{L}_{X} \alpha=\operatorname{di}_{X} \alpha+\mathbf{i}_{X} \mathbf{d} \alpha
$$

This is proved by straightforward calculation.

\subsection{Symplectic manifolds; the cotangent bundle as a symplec- tic manifold}

Any cotangent bundle $T^{*} Q$ has a natural symplectic structure, which is the geometric structure on manifolds corresponding to the symplectic matrix $\omega$ introduced by eq. 4.10, and to the symplectic forms on vector spaces defined at the end of Section 4.3.3. (Here 'natural' means intrinsic, and in particular, independent of a choice of coordinates or bases.) It is this structure that enables a scalar function to determine a dynamics. 
That is: the symplectic structure implies that any scalar function $H: T^{*} Q \rightarrow \mathbb{R}$ defines a vector field $X_{H}$ on $T^{*} Q$.

I first describe this structure (Section 6.3.1), and then show that any cotangent bundle has it (Section 6.3.2). Later subsections will develop the consequences.

\subsubsection{Symplectic manifolds}

A symplectic structure or symplectic form on a manifold $M$ is defined to be a differential 2 -form $\omega$ on $M$ that is closed (i.e. $\mathbf{d} \omega=0$ ) and non-degenerate. That is: for any $x \in M$, and any two tangent vectors at $x, \sigma, \tau \in T_{x}$ :

$$
\mathbf{d} \omega=0 \text { and } \forall \tau \neq 0, \quad \exists \sigma: \quad \omega(\tau, \sigma) \neq 0 .
$$

Such a pair $(M, \omega)$ is called a symplectic manifold.

There is a rich theory of symplectic manifolds; but we shall only need a small fragment of it, building on our discussion in Section 4.3.3. (In particular, the fact that we mostly avoid the theory of canonical transformations means we will not need the theory of Lagrangian sub-manifolds.)

First, it follows from the non-degeneracy of $\omega$ that $M$ is even-dimensional; (cf. eq. 4.38).

It also follows that at any $x \in M$, there is a basis-independent isomorphism $\omega^{b}$ from the tangent space $T_{x}$ to its dual $T_{x}^{*}$. We saw this in (2) and (4) of Section 4.3.3, especially eq. 4.23. Namely: for any $x \in M$ and $\tau \in T_{x}$, the value of the 1 -form $\omega^{b}(\tau) \in T_{x}^{*}$ is defined by

$$
\omega^{b}(\tau)(\sigma):=\omega(\sigma, \tau) \quad \forall \sigma \in T_{x} .
$$

Here we return to the main idea emphasised already in Section 4.3.1: that symplectic structure enables a covector field, i.e. a differential one-form, to determine a vector field. Thus for any function $H: M \rightarrow \mathbb{R}$, so that $d H$ is a differential 1 -form on $M$, the inverse of $\omega^{b}$ (which we might write as $\omega^{\sharp}$ ), carries $d H$ to a vector field on $M$, written $X_{H}$. Cf. eq. 4.14 .

So far, we have noted some implications of $\omega$ being non-degenerate. The other part of the definition of a symplectic form (for a manifold), viz. $\omega$ being closed, $\mathbf{d} \omega=0$, is also important. We shall see in Section 6.5 that it implies that a vector field $X$ on a symplectic manifold $M$ preserves the symplectic form $\omega$ (i.e. in more physical jargon: generates (a one-parameter family of) canonical transformations) iff $X$ is Hamiltonian in the sense of Section 5.2; i.e. there is a scalar function $f$ such that $X=X_{f} \equiv \omega^{\sharp}(d f)$. Or in terms of the Poisson bracket, with - representing the argument place for a scalar function: $X(\cdot)=X_{f}(\cdot) \equiv\{\cdot, f\}$.

So much by way of introducing symplectic manifolds. I turn to showing that any cotangent bundle $T^{*} Q$ is such a manifold. 


\subsubsection{The cotangent bundle}

Choose any local coordinates $q$ on $Q(\operatorname{dim}(Q)=n)$, and the natural local coordinates $q, p$ thereby induced on $T^{*} Q$; (cf. (B) of Section 6.1). We define the 2-form

$$
d p \wedge d q:=d p_{i} \wedge d q^{i}:=\sum_{i=1}^{n} d p_{i} \wedge d q^{i} .
$$

To show that eq. 6.14 defines the same 2 -form, whatever choice we make of the chart $q$ on $Q$, it suffices to show that $d p \wedge d q$ is the exterior derivative of a 1 -form on $T^{*} Q$ which is defined naturally (i.e. independently of coordinates or bases) from the derivative (also known as: tangent) map of the projection

$$
\pi:(q, p) \in T^{*} Q \mapsto q \in Q .
$$

Thus consider a tangent vector $\tau$ (not to $Q$, but) to the cotangent bundle $T^{*} Q$ at a point $\eta=(q, p) \in T^{*} Q$, i.e. $q \in Q$ and $p \in T_{q}^{*}$. Let us write this as: $\tau \in T_{\eta}\left(T^{*} Q\right) \equiv$ $T_{(q, p)}\left(T^{*} Q\right)$. The derivative map, $D \pi$ say, of the natural projection $\pi$ applies to $\tau$ :

$$
D \pi: \tau \in T_{(q, p)}\left(T^{*} Q\right) \mapsto(D \pi(\tau)) \in T_{q} .
$$

Now define a 1 -form $\theta_{H}$ on $T^{*} Q$ by

$$
\theta_{H}: \tau \in T_{(q, p)}\left(T^{*} Q\right) \mapsto p(D \pi(\tau)) \in \mathbb{R} ;
$$

where in this definition of $\theta_{H}, p$ is defined to be the second component of $\tau$ 's base-point $(q, p) \in T^{*} Q$; i.e. $\tau \in T_{(q, p)}\left(T^{*} Q\right)$ and $p \in T_{q}^{*}$.

This 1 -form is called the canonical 1-form on $T^{*} Q$. It is the "Hamiltonian version" of the 1-form $\theta_{L}$ defined by eq. 2.13; and also there called the 'canonical 1-form'. But Section 6.1's discussion of the "fruitful ambiguity" of the symbol $p$ brings out

a contrast. While $\theta_{L}$ as defined by eq. 2.13 clearly depends on $L$, the definition of $\theta_{H}$, eq. 6.17, does not depend on any function $H \cdot \theta_{H}$ is given just by the cotangent bundle structure. Hence the subscript $H$ here just indicates "Hamiltonian (as against Lagrangian) version", not dependence on a function $H$.

So much by way of a natural definition of a 1-form. One now checks that in any natural local coordinates $q, p, \theta_{H}$ is given by

$$
\theta_{H}=p_{i} d q^{i}
$$

Finally, we define a 2-form by taking the exterior derivative of $\theta_{H}$ :

$$
\mathbf{d}\left(\theta_{H}\right):=\mathbf{d}\left(p_{i} d q^{i}\right) \equiv d p_{i} \wedge d q^{i} .
$$

where the last equation follows immediately from eq. 6.10 . One checks that this 2 -form is closed (since $\mathbf{d}^{2}=0$ ) and non-degenerate. So $\left(T^{*} Q, \mathbf{d}\left(\theta_{H}\right)\right)$ is a symplectic manifold.

Referring to eq. 4.18 of Section 4.3, or eq. 4.39 of Section 4.3.3, or eq. 6.3 of Section 6.2 , we see that at each point $(q, p) \in T^{*} Q$, this symplectic form is, upto a sign, our familiar "sum of signed areas" - first seen as induced by the matrix $\omega$ of eq. 4.10. 
Accordingly, Section 4.3.3's definition of a canonical symplectic form is extended to the present case: $\mathbf{d}\left(\theta_{H}\right)$, or its negative $-\mathbf{d}\left(\theta_{H}\right)$, is called the canonical symplectic form, or canonical 2-form. (The difference from Section 4.3.3's definition is that on a manifold, the symplectic form is required to be closed.)

(The difference by a sign is of course conventional: it arises from our taking the $q \mathrm{~s}$, not the $p \mathrm{~s}$, as the first $n$ out of the $2 n$ coordinates. For if we had instead taken the $p$ s, the matrix occurring in eq. 4.12 would have been $-\omega \equiv \omega^{-1}$ : exactly matching the cotangent bundle's intrinsic 2 -form $\mathbf{d}\left(\theta_{H}\right)$.)

We will see, in Section 6.6, a theorem (Darboux's theorem) to the effect that locally, any symplectic manifold "looks like" a cotangent bundle: or in other words, a cotangent bundle is locally a "universal" example of symplectic structure. But first we return, in the next two Subsections, to Hamilton's equations, and Noether's theorem.

\subsection{Geometric formulations of Hamilton's equations}

We already emphasised in Sections 4.3 and 5 the main geometric idea behind Hamilton's equations: that a gradient, i.e. covector, field $d H$ determines a vector field $X_{H}$. We first saw this determination via the symplectic matrix, in eq. 4.14 of Section 4.3.1, viz.

$$
X_{H}(z)=\omega \nabla H(z) ;
$$

and then via the Poisson bracket, in eq. 5.14 of Section 5.2, viz.

$$
D:=X_{H}=\frac{d}{d t}=\dot{q}^{i} \frac{\partial}{\partial q^{i}}+\dot{p}_{i} \frac{\partial}{\partial p_{i}}=\frac{\partial H}{\partial p_{i}} \frac{\partial}{\partial q^{i}}-\frac{\partial H}{\partial q^{i}} \frac{\partial}{\partial p_{i}}=\{\cdot, H\} .
$$

The symplectic structure and Poisson bracket were related by eq. 5.7, viz.

$$
\{f, g\}(z)=\tilde{\nabla} f(z) \cdot \omega \cdot \nabla g(z) .
$$

And to this earlier discussion, the last Subsection, Section 6.3, added the identification of the canonical symplectic form of a cotangent bundle, eq. 6.19.

Let us sum up these discussions by giving some geometric formulations of Hamilton's equations at a point $z=(q, p)$ in a cotangent bundle $T^{*} Q$. Let us write $\omega^{\sharp}$ for the (basis-independent) isomorphism from the cotangent space to the tangent space, $T_{z}^{*} \rightarrow T_{z}$, induced by $\omega:=-\mathbf{d}\left(\theta_{H}\right)=d q^{i} \wedge d p_{i}$ (cf. eq. 4.35 and 6.13). Then Hamilton's equations, eq. 4.14 or 6.20 , may be written as:

$$
\dot{z}=X_{H}(z)=\omega^{\sharp}(\mathbf{d} H(z))=\omega^{\sharp}(d H(z)) .
$$

Applying $\omega^{b}$, the inverse isomorphism $T_{z} \rightarrow T_{z}^{*}$, to both sides, we get

$$
\omega^{b} X_{H}(z)=d H(z) \text {. }
$$

In terms of the symplectic form $\omega$ at $z$, this is (cf. eq. 4.23): for all vectors $\tau \in T_{z}$

$$
\omega\left(X_{H}(z), \tau\right)=d H(z) \cdot \tau ;
$$


or in terms of the contraction defined by eq. 6.4 , with $\cdot$ marking the argument place of $\tau \in T_{z}$ :

$$
\mathbf{i}_{X_{H}} \omega:=\omega\left(X_{H}(z), \cdot\right)=d H(z)(\cdot) .
$$

More briefly, and now for any function $f$, it is:

$$
\mathbf{i}_{X_{f}} \omega=d f .
$$

Here is a final example. Recall the relation between the Poisson bracket and the directional derivative (or the Lie derivative $\mathcal{L}$ ) of a function, eq. 5.15 and 6.21: viz.

$$
\mathcal{L}_{X_{f}} g=d g\left(X_{f}\right)=X_{f}(g)=\{g, f\} .
$$

Combining this with eq. 6.27 , we can reformulate the relation between the symplectic form and Poisson bracket, eq. 6.22, in the form:

$$
\{g, f\}=d g\left(X_{f}\right)=\mathbf{i}_{X_{f}} d g=\mathbf{i}_{X_{f}}\left(\mathbf{i}_{X_{g}} \omega\right)=\omega\left(X_{g}, X_{f}\right) .
$$

\subsection{Noether's theorem completed}

The discussion of Noether's theorem in Section 5.3 left unfinished business: to prove that a vector field generates a one-parameter family of canonical transformations iff it is a Hamiltonian vector field (and so justify the third claim of Section 5.3.1). Cartan's magic formula and the Poincaré Lemma, both from Section 6.2, make it easy to prove this, for a vector field on any symplectic manifold $(M, \omega) . \quad((M, \omega)$ need not be a cotangent bundle.)

We define a vector field $X$ on a symplectic manifold $(M, \omega)$ to be symplectic (also known as: canonical) iff the Lie-derivative along $X$ of the symplectic form vanishes, i.e. $\mathcal{L}_{X} \omega=0 .{ }^{18}$

Since $\omega$ is closed, i.e. $\mathbf{d} \omega=0$, Cartan's magic formula, eq. 6.11, applied to $\omega$ becomes

$$
\mathcal{L}_{X} \omega \equiv \mathbf{d i}_{X} \omega+\mathbf{i}_{X} \mathbf{d} \omega=\operatorname{di}_{X} \omega
$$

So for $X$ to be symplectic is for $\mathbf{i}_{X} \omega$ to be closed. But by the Poincaré Lemma, if $\mathbf{i}_{X} \omega$ is closed, it is locally exact. That is: there locally exists a scalar function $f: M \rightarrow \mathbb{R}$ such that

$$
\mathbf{i}_{X} \omega=d f \text { i.e. } X=X_{f} .
$$

So for $X$ to be symplectic is equivalent to $X$ being locally Hamiltonian.

\footnotetext{
${ }^{18}$ As announced in Section 2.2.1, I assume the notion of the Lie-derivative, in particular the Liederivative of a 2 -form. Suffice it to say, as a sketch, that the flow of $X$ defines a map on $M$ which induces a map on curves, and so on vectors, and so on co-vectors, and so on 2-forms such as $\omega$. Nor will I go into details about the equivalence between this definition of $X$ 's being symplectic, and $X$ 's generating (active) canonical transformations, or preserving the Poisson bracket. For as I have emphasised, I will not need to develop the theory of canonical transformations.
} 
So we can sum up Noether's theorem from a geometric perspective, as follows. We define a Hamilton system to be a triple $(M, \omega, H)$ where $(M, \omega)$ is a symplectic manifold and $H: M \rightarrow \mathbb{R}$, i.e. $M \in \mathcal{F}(M)$. We define a (continuous) symmetry of a Hamiltonian system to be a vector field $X$ on $M$ that preserves both the symplectic form, $\mathcal{L}_{X} \omega=0$, and the Hamiltonian function, $\mathcal{L}_{X} H=0$. As we have just seen: for any symmetry so defined, there locally exists an $f$ such that $X=X_{f}$. So we can apply the "one-liner", eq. 5.18, i.e. the antisymmetry of the Poisson bracket,

$$
X_{f}(H) \equiv\{H, f\}=0 \quad \text { iff } \quad X_{H}(f) \equiv\{f, H\}=0,
$$

to conclude that $f$ is a first integral (constant of the motion). Thus we have

Noether's theorem for a Hamilton system If $X$ is a symmetry of a Hamiltonian system $(M, \omega, H)$, then locally $X=X_{f}$ and $f$ is a constant of the motion. And conversely: if $f: M \rightarrow \mathbb{R}$ is a constant of the motion, then $X_{f}$ is a symmetry. Besides, this result encompasses the Lagrangian version of the theorem; cf. Sections 3.4 and 5.3.

Example:- For most Hamiltonian systems in euclidean space $\mathbb{R}^{3}$, spatial translations and rotations are (continuous) symmetries. For example, consider $N$ pointparticles interacting by Newtonian gravity. The Hamiltonian is a sum of two terms, which are each individually invariant under these euclidean motions:

(i) a kinetic energy term $K$; though I will not go into details, it is in fact defined by the euclidean metric of $\mathbb{R}^{3}$ (cf. footnote 4 in Section 2.1), and is thereby invariant; and

(ii) a potential energy term $V$; it depends only on the particles' relative distances, and is thereby invariant.

The corresponding conserved quantities are the total linear and angular momentum. $^{19}$

Finally, an incidental remark which relates to the "rectification theorem", that on any manifold any vector field $X$ can be "straightened out" in a neighbourhood around any point at which $X$ is non-zero, so as to have all but one component vanish and the last component equal to 1 ; cf. eq. 3.22. Using this theorem, it is easy to see that on any even-dimensional manifold any vector field $X$ is locally Hamiltonian, with respect to some symplectic form, around a point where $X$ is non-zero. (One defines the symplectic form by Lie-dragging from a surface transverse to $X$ 's integral curves.)

\subsection{Darboux's theorem, and its role in reduction}

Darboux's theorem states that cotangent bundles are, locally, a "universal form" of symplectic manifold. That is: Not only is any symplectic manifold $(M, \omega)$ evendimensional. Also, it "looks locally like" a cotangent bundle, in that around any $x$

\footnotetext{
${ }^{19}$ By the way, this Hamiltonian is not invariant under boosts. But as I said in Section 2.2.1 and footnote 8 , I restrict myself to time-independent transformations; the treatment of symmetries that "represent the relativity of motion" needs separate discussion.
} 
in $M$, there is a local coordinate system $\left(q^{1}, \ldots, q^{n} ; p_{1}, \ldots, p_{n}\right)$-where the use of both upper and lower indices is now just conventional, with no meaning about dual bases!in which:

(i) $\omega$ takes the form $d q^{i} \wedge d p_{i}$; and so

(ii) the Poisson brackets of the $q$ s and $p$ s take the fundamental form in eq. 5.13. (The theorem generalizes to the Poisson manifolds mentioned in Section 6.8.)

Besides, the proof of Darboux's theorem yields further information: information which is important for reducing problems. It arises from the beginning of the proof; and will return us to Section 4.2's point that the elementary connection between cyclic coordinates and conserved conjugate momenta underpins the role of symmetries and conserved quantities in reductions on symplectic manifolds.

(In fact, Darboux's theorem also yields two other broad implications about reducing problems; but I will not develop the details here. The second implication concerns the way that a Hamiltonian structure is preserved in the reduced problem. The third implication concerns the requirement that constants of the motion be in involution, i.e. have vanishing Poisson bracket with each other; so it leads to the idea of complete integrability - a topic this paper foreswears.)

Namely, the proof implies that "almost" any scalar function $f \in \mathcal{F}(M)$ can be taken as the first "momentum" coordinate $p_{1}$; or as the first configurational coordinate $q^{1}$. Here "almost" is not meant in a measure-theoretic sense; it is just that $f$ is subject to a mild restriction, that $d f \neq 0$ at the point $x \in M$.

In a bit more detail: The proof of Darboux's theorem starts by taking any such $f$ to be our $p_{1}$, and then constructs the canonically conjugate generalized coordinate $q^{1}$, i.e. the coordinate such that $\left\{q^{1}, p_{1}\right\}=1$ : so that $p_{1}$ generates translation in the direction of increasing $q^{1}$. Indeed the construction is geometrically clear. The symplectic structure means that any such $f$ defines a Hamiltonian vector field $X_{f}$, and a flow $\phi^{f}$. We choose a $(2 n-1)$-dimensional local submanifold $N$ passing through the given point $x$, and transverse to all the integral curves of $X_{f}$ in a neighbourhood of $x$; and we set the parameter $\lambda$ of the flow $\phi^{f}$ to be zero at all points $y \in N$. Then for any $z$ in a suitably small neighbourhood of the given point $x$, we define the function $q^{1}(z)$ to be the parameter-value at $z$ of the integral curve of $X_{f}$ that passes through $z$. So by construction, (i) $f$ generates translation in the direction of increasing $q^{1}$, and (ii) defining $p_{1}:=f$, we have $\left\{q^{1}, p_{1}\right\}=1$.

This is just the beginning of the proof. But I will not need details of how it goes on to establish the local existence of canonical coordinates, i.e. coordinates such that analogues of (i) and (ii), also for $i \neq 1$, hold. In short, the strategy is to use induction on the dimension of the manifold; for details, cf. e.g. Arnold (1989: 230-232).

To see the significance of this for reducing problems, suppose that there is a constant of the motion, and that we take it as our $f$, i.e. as the first momentum coordinate $p_{1}$. So the system evolves on a $(2 n-1)$-dimensional manifold given by an equation $f=$ constant. So writing $H$ in the canonical coordinate system secured by Darboux's theorem, we conclude that $0=\dot{f} \equiv-\frac{\partial H}{\partial q^{1}}$. That is, $q^{1}$ is cyclic. So as discussed in 
Section 4.2 , we need only solve the problem in the $2 n-2$ variables $q^{2}, \ldots, q^{n} ; p_{2}, \ldots, p_{n}$. Having done so, we can find $q^{1}$ as a function of time, by solving eq. 4.9 by quadrature.

To put the point in geometric terms:-

(i): The system is confined to a $(2 n-1)$-dimensional manifold $p_{1}=\alpha=$ constant, $M_{\alpha}$ say.

(ii): $M_{\alpha}$ is foliated by a local one-parameter family of $(2 n-2)$-dimensional manifolds labelled by values of $q^{1} \in I \subset \mathbb{R}, M_{\alpha}=\cup_{q^{1} \in I} M_{\alpha, q^{1}}$.

(iii): Of course, the dynamical vector field is transverse to the leaves of this foliation; i.e. $q^{1}$ is not a constant of the motion, $\dot{q}^{1} \neq 0$. But since $q^{1}$ is ignorable, $\frac{\partial H}{\partial q^{1}}=0$, the problem to be solved is "the same" at points $x_{1}, x_{2}$ that differ only in their values of $q^{1}$.

\subsection{Geometric formulation of the Legendre transformation}

Let us round off our development of both Lagrangian and Hamiltonian mechanics, by formulating the Legendre transformation as a map from the tangent bundle $T Q$ to the cotangent bundle $T^{*} Q$. In this formulation, the Legendre transformation is often called the fibre derivative.

Again, there is a rich theory to be had here. In part, it relates to the topics mentioned in Section 4.2.3: (i) the description of a function (in the simplest case $f: \mathbb{R} \rightarrow \mathbb{R}$ ) by its gradients and axis-intercepts, rather than by its arguments and values; (ii) variational principles. But I shall not go into details about this theory: since this paper emphasises the Hamiltonian framework, a mere glimpse of this theory must suffice. (References, additional to those in Section 4.2.3, include: Abraham and Marsden (1978: Sections 3.6-3.8) and Marsden and Ratiu (1999: Sections 7.2-7.5, 8.18.3).)

Let us return to the Lagrangian framework. We stressed in Section 2.2 that a scalar on the tangent bundle, the Lagrangian $L: T Q \rightarrow \mathbb{R}$, "determines everything": the dynamical vector field $D=: D_{L}$; and so for given initial $q$ and $\dot{q}, L$ determines a solution, a trajectory in $T Q$, i.e. $2 n$ functions of time $q(t), \dot{q}(t)$ with the first $n$ functions determining the latter.

For the Legendre transformation, the fundamental points are that:

(1): $L$ also determines at any point $q \in Q$, a preferred map $F L_{q}$ from the tangent space $T_{q}$ to its dual space $T_{q}^{*}$. Besides this preferred map:

(2): extends trivially to a preferred map from all of $T Q$ to $T^{*} Q$; this is the Legendre transformation, understood geometrically;

(3): extends, under some technical conditions (about certain kinds of uniqueness, invertibility and smoothness), so as to carry geometric objects of various sorts defined on $T Q$ to corresponding objects defined on $T^{*} Q$, and vice versa.

So under these conditions, the Legendre transformation (together with its inverse) transfers the entire description of the system's motion between the Lagrangian and Hamiltonian frameworks. 
I will explain (1) and (2), but just gesture at (3).

(1): Intuitively, the preferred map $F L_{q}$ from each tangent space $T_{q}$ to its dual space $T_{q}^{*}$ is the transition $\dot{q} \mapsto p$. More precisely: since $L$ is a scalar on $T Q$, any choice of local coordinates $q$ on a patch of $Q$, together with the induced local coordinates $q, \dot{q}$ on a patch of $T Q$, defines the partial derivatives $\frac{\partial L}{\partial \dot{q}}$. At any point $q$ in the domain of the local coordinates, this defines a preferred map $F L_{q}$ from the tangent space $T_{q}$ to the dual space $T_{q}^{*}: F L_{q}: T_{q} \rightarrow T_{q}^{*}$. Namely, a vector $\tau \in T_{q}$ with components $\dot{q}^{i}$ in the coordinate system $q^{i}$ on $Q$, i.e. $\tau=\dot{q}^{i} \frac{\partial}{\partial q^{i}}$ (think of a motion through configuration $q$ with generalized velocity $\tau$ ) is mapped to the 1 -form whose components in the dual basis $d q^{i}$ are $\frac{\partial L}{\partial \dot{q}^{i}}$. That is

$$
F L_{q}: \tau=\dot{q}^{i} \frac{\partial}{\partial q^{i}} \in T_{q} \mapsto \frac{\partial L}{\partial \dot{q}^{i}} d q^{i} \in T_{q}^{*}
$$

One easily checks that because the canonical momenta are a 1-form, this definition is, despite appearances, coordinate-independent.

(2): An equivalent definition, manifestly coordinate-independent and given for all $q \in Q$, is as follows. Given $L: T Q \rightarrow \mathbb{R}$, define $F L: T Q \rightarrow T^{*} Q$, the fibre derivative, by

$$
\forall q \in Q, \forall \sigma, \tau \in T_{q}: F L(\sigma) \cdot \tau=\left.\frac{d}{d s}\right|_{s=0} L(\sigma+s \tau)
$$

(We here take $\sigma, \tau$ to encode the identity of the base-point $q$, so that we make notation simpler, writing $F L(\sigma)$ rather than $F L((q, \sigma))$ etc.) That is: $F L(\sigma) \cdot \tau$ is the derivative of $L$ at $\sigma$, along the fibre $T_{q}$ of the fibre bundle $T Q$, in the direction $\tau$. So $F L$ is fibrepreserving: i.e. it maps the fibre $T_{q}$ of $T Q$ to the fibre $T_{q}^{*}$ of $T^{*} Q$. In local coordinates $q, \dot{q}$ on $T Q, F L$ is given by:

$$
F L\left(q^{i}, \dot{q}^{i}\right)=\left(q^{i}, \frac{\partial L}{\partial \dot{q}^{i}}\right) ; \text { i.e. } p_{i}=\frac{\partial L}{\partial \dot{q}^{i}} .
$$

An important special case involves a free system (i.e. no potential term in the Lagrangian) and a configuration manifold $Q$ with a metric $g=g_{i j}$ defined by the kinetic energy. (Cf. footnote 4 for the definition of this metric: in short, the constraints being scleronomous (i.e. time-independent, cf. Section 2.1), implies that for any coordinate system on $Q$, the kinetic energy is a homogeneous quadratic form in the generalized velocities.) The Lagrangian is then just the kinetic energy of the metric,

$$
L(q, \dot{q}) \equiv L(\dot{q}):=\frac{1}{2} g_{i j} \dot{q}^{i} \dot{q}^{j}
$$

so that the fibre derivative is given by

$$
F L(\sigma) \cdot \tau=g(\sigma, \tau)=g_{i j} \sigma^{i} \tau^{j} \quad \text {, i.e. } \quad p_{i}=g_{i j} \dot{q}^{j} .
$$

(3): We can use $F L$ to pull-back to $T Q$ the canonical 1 -form $\theta \equiv \theta_{H}$ and symplectic form $\omega$ from $T^{*} Q$ (eq. 6.17 and 6.18 with $\omega=-\mathbf{d} \theta$, from Section 6.3.B). That is, we 
can define

$$
\theta_{L}:=(F L)^{*} \theta_{H} \text { and } \omega_{L}:=(F L)^{*} \omega .
$$

Since exterior differentiation $\mathbf{d}$ commutes with pull-backs, $\omega_{L}=-\mathbf{d} \theta_{L}$. Furthermore:

(i): As one would hope, $\theta_{L}$, so defined, is Lagrangian mechanics' canonical 1-form, which we already defined in eq. 2.13 (and which played a central role in the Lagrangian version of Noether's theorem).

(ii): One can show that $\omega_{L}$ is non-degenerate iff the Hessian condition eq. 2.3 holds. So under this condition, we can analyse Lagrangian mechanics in terms of symplectic structure.

Given $L$, we define its energy function $E: T Q \rightarrow \mathbb{R}$ by

$$
\forall v \equiv(q, \tau) \in T Q, \quad E(v):=F L(v) \cdot v-L(v) ;
$$

or in coordinates

$$
E\left(q^{i}, \dot{q}^{i}\right):=\frac{\partial L}{\partial \dot{q}^{i}} \dot{q}^{i}-L\left(q^{i}, \dot{q}^{i}\right)
$$

If $F L$ is a diffeomorphism, we find that $E \circ(F L)^{-1}$ is, as one would hope, the Hamiltonian function $H: T^{*} Q \rightarrow \mathbb{R}$ which we already defined in eq. 4.4 .

And accordingly, if $F L$ is a diffeomorphism, then the derivative of $F L$ carries the dynamical vector field $\frac{d}{d t}$ in the Lagrangian description, as defined in eq. 2.8 (Section $2.2,(2))$, viz.

$$
D_{L}:=\dot{q}^{i} \frac{\partial}{\partial q^{i}}+\ddot{q}^{i} \frac{\partial}{\partial \dot{q}^{i}}
$$

to the Hamiltonian dynamical vector field, viz.

$$
D_{H}:=\dot{q}^{i} \frac{\partial}{\partial q^{i}}+\dot{p}_{i} \frac{\partial}{\partial p_{i}}
$$

More generally, one can show if $F L$ is a diffeomorphism, there is a bijective correspondence between the various geometric structures used in the Lagrangian and Hamiltonian descriptions. For precise statements of this idea, cf. e.g. Abraham and Marsden (1978: Theorem 3.6.9) and Marsden and Ratiu (1999: Theorem 7.4.3.), and their preceding discussions.

\subsection{Glimpsing the more general framework of Poisson mani- folds}

Recall that Section 5.1 listed several properties of the Poisson bracket, as defined by eq. 5.3 or 5.6. We end by briefly describing how the postulation of a bracket that acts on the scalar functions $F: M \rightarrow \mathbb{R}$ defined on any manifold $M$, and possesses four of Section 5.1's listed properties, provides a sufficient framework for mechanics in Hamiltonian style. The bracket is again called a 'Poisson bracket', and the manifold $M$ equipped with such a bracket is called a Poisson manifold. 
Namely, we require the following four properties. The Poisson bracket is to be bilinear; antisymmetric; and to obey the Jacobi identity (eq. 5.11) for any real functions $F, G, H$ on $M$, i.e.

$$
\{\{F, H\}, G\}+\{\{G, F\}, H\}+\{\{H, G\}, F\}=0 ;
$$

and to obey Leibniz' rule for products (eq. 5.9), i.e.

$$
\{F, H \cdot G\}=\{F, H\} \cdot G+H \cdot\{F, G\}
$$

This generalizes Hamiltonian mechanics: in particular, a Poisson manifold need not be a symplectic manifold. The main idea of the extra generality is that the antisymmetric bilinear map that gives the geometry of the state space (the analogue of Section 4.3's symplectic form $\omega$ ) can be degenerate. So this map can "have extra zeroes", as in eq. 4.37 and 4.38. (This map is induced by the generalized Poisson bracket, via an analogue of eq. 5.7.) This means that a Poisson manifold can have odd dimension; while we saw in Section 4.3.3 that any symplectic vector space is even-dimensional - and so, therefore, is any symplectic manifold (Section 6.3.1 and 6.6).

On the other hand, the generalized framework has strong connections with the usual one. ${ }^{20}$ One main connection is the result that any Poisson manifold $M$ is a disjoint union of even-dimensional manifolds, on each of which $M$ 's degenerate antisymmetric bilinear form (induced by the generalized Poisson bracket) restricts to be non-degenerate; so that there is an orthodox Hamiltonian mechanics on each such 'symplectic leaf'. Another main connection is that Section 5.3's "one-liner" version of Noether's theorem, eq. 5.18, underpins versions of Noether's theorem for the more general framework.

This generalized framework is important for various reasons; I will just mention two.

(i): For a system whose orthodox Hamiltonian mechanics on a symplectic manifold (dimension $2 n$, say) depends on $s$ real parameters, it is sometimes natural to consider the corresponding $(2 n+s)$-dimensional space. This is often a Poisson manifold; viz., one foliated into an $s$-dimensional family of $2 n$-dimensional symplectic manifolds. This scenario occurs even for some very familiar systems, such as the pivoted rigid body described by Euler's equations.

(ii): Poisson manifolds often arise in the theory of symplectic reduction. For when you quotient a symplectic manifold by the action of a group (e.g. a group of symmetries of a Hamiltonian system in the sense of Section 6.5), you often get a Poisson manifold, rather than a symplectic one. Indeed, the pivoted rigid body is itself an example of this.

But this generalized framework is a large topic, which we cannot go into: as mentioned, Butterfield (2006) is a philosopher's introduction.

\footnotetext{
${ }^{20}$ Because of these connections, it is natural to still call the more general framework 'Hamiltonian'; as is usually done. But of course this is just a verbal matter.
} 
For now, we end with a historical point. ${ }^{21}$ It is humbling, but also I hope inspiring, reflection about one of classical mechanics' monumental figures. Namely: a considerable part of the modern theory of Poisson manifolds, including their uses for the rigid body and for symplectic reduction, was already contained in Lie (1890)!

Acknowledgements:- I am grateful to the editors, not least for their patience; to audiences in Irvine, Oxford, Princeton and Santa Barbara; and to Katherine Brading, Harvey Brown, Hans Halvorson, David Malament, Wayne Myrvold, David Wallace, and especially Graeme Segal, for conversations, comments-and corrections!

\section{$7 \quad$ References}

R. Abraham and J. Marsden (1978), Foundations of Mechanics, second edition: AddisonWesley.

V. Arnold (1973), Ordinary Differential Equations, MIT Press.

V. Arnold (1989), Mathematical Methods of Classical Mechanics, Springer, (second edition).

G. Belot (2003), 'Notes on symmetries', in Brading and Castellani (ed.s) (2003), pp. 393-412.

K. Brading and E. Castellani (ed.s) (2003), Symmetry in Physics, Cambridge University Press.

H. Brown and P. Holland (2004), 'Simple applications of Noether's first theorem in quantum mechanics and electromagnetism', American Journal of Physics $\mathbf{7 2}$ p. 34-39. Available at: http://arxiv.org/abs/quant-ph/0302062 and http://philsciarchive.pitt.edu/archive/00000995/

H. Brown and P. Holland (2004a), 'Dynamical vs. variational symmetries: Understanding Noether's first theorem', Molecular Physics, 102, (11-12 Special Issue), pp. 1133-1139.

J. Butterfield (2004), 'Some Aspects of Modality in Analytical mechanics', in Formal Teleology and Causality, ed. M. Stöltzner, P. Weingartner, Paderborn: Mentis. Available at Los Alamos arXive: http://arxiv.org/abs/physics/0210081 or http://xxx.soton.ac.uk/abs/physics/0210081; and at Pittsburgh archive: http://philsciarchive.pitt.edu/archive/00001192.

J. Butterfield (2004a), 'Between Laws and Models: Some Philosophical Morals of Lagrangian Mechanics'; available at Los Alamos arXive: http://arxiv.org/abs/physics/0409030 or http://xxx.soton.ac.uk/abs/physics/0409030; and at Pittsburgh archive: http://philsci-

\footnotetext{
${ }^{21}$ As mentioned in footnote 10, Olver (2000) gives many details especially about Lie; e.g. Olver (2000: 374-379, 427-428). Cf. also Marsden and Ratiu (1999: 336-338, 430-432), and for a full history, Hawkins (2000).
} 
archive.pitt.edu/archive/00001937/.

J. Butterfield (2004b), 'On Hamilton-Jacobi Theory as a Classical Root of Theory', in A. Elitzur, S. Dolev and N. Kolenda (eds.), Quo Vadis Quantum Mechanics?, Springer, pp. 239-273; available at Los Alamos arXive: http://arxiv.org/abs/quantph/0210140; or at Pittsburgh archive: http://philsci-archive.pitt.edu/archive/00001193/

J. Butterfield (2005), 'Between Laws and Models: Some Philosophical Morals of Hamiltonian Mechanics', in preparation.

J. Butterfield (2006), 'On Symplectic Reduction in Classical Mechanics', forthcoming in The North Holland Handbook of Philosophy of Physics, ed. J. Earman and J. Butterfield, North Holland.

R. Courant and D. Hilbert (1953), Methods of Mathematical Physics, volume I, Wiley-Interscience (Wiley Classics 1989).

R. Courant and D. Hilbert (1962), Methods of Mathematical Physics, volume II, Wiley-Interscience (Wiley Classics 1989).

E. Desloge (1982), Classical Mechanics, John Wiley.

J. Earman (2003), 'Tracking down gauge: an ode to the constrained Hamiltonian formalism', in Brading and Castellani (ed.s) (2003), pp. 140-162.

H. Goldstein et al. (2002), Classical Mechanics, Addison-Wesley, (third edition).

T. Hawkins (2000), Emergence of the Theory of Lie Groups: an essay in the history of mathematics 1869-1926, New York: Springer.

M. Henneaux and C. Teitelboim (1992), Quantization of Gauge Systems, Princeton University Press.

O. Johns (2005), Analytical Mechanics for Relativity and Quantum Mechanics, Oxford University Press, forthcoming.

J. José and E. Saletan (1998), Classical Dynamics: a Contemporary Approach, Cambridge University Press.

H. Kastrup (1987), 'The contributions of Emmy Noether, Felix Klein and Sophus Lie to the modern concept of symmetries in physical systems', in Symmetries in Physics (1600-1980), Barcelona: Bellaterra, Universitat Autonoma de Barcelona, p. 113-163.

S. Lie (1890). Theorie der Transformationsgruppen: zweiter abschnitt, Leipzig: B.G.Teubner.

C. Lanczos (1986), The Variational Principles of Mechanics, Dover; (reprint of the 4th edition of 1970).

J. Marsden and T. Ratiu (1999), Introduction to Mechanics and Symmetry, second edition: Springer-Verlag.

G. Morandi et al (1990), 'The inverse problem of the calculus of variations and the geometry of the tangent bundle', Physics Reports 188, p. 147-284. 
P. Olver (2000), Applications of Lie Groups to Differential Equations, second edition: Springer-Verlag.

D. Wallace (2003), 'Time-dependent Symmetries: the link between gauge symmetries and indeterminism', in Brading and Castellani (ed.s) (2003), pp. 163-173.

E. Wigner (1954), 'Conservation laws in classical and quantum physics', Progress of Theoretical Physics 11, p. 437-440. 